\title{
Traveling Wave Solutions for Epidemic Cholera Model with Disease-Related Death
}

\author{
Tianran Zhang ${ }^{1}$ and Qingming Gou ${ }^{2}$ \\ ${ }^{1}$ School of Mathematics and Statistics, Southwest University, Chongqing 400715, China \\ ${ }^{2}$ College of Mathematics \& Computer Science, Yangtze Normal University, Chongqing 408100, China \\ Correspondence should be addressed to Tianran Zhang; zhtr0123@126.com
}

Received 2 January 2014; Accepted 10 March 2014; Published 27 April 2014

Academic Editors: M. Han, Z. Jin, and Y. Xia

Copyright (C) 2014 T. Zhang and Q. Gou. This is an open access article distributed under the Creative Commons Attribution License, which permits unrestricted use, distribution, and reproduction in any medium, provided the original work is properly cited.

Based on Codeço's cholera model (2001), an epidemic cholera model that incorporates the pathogen diffusion and disease-related death is proposed. The formula for minimal wave speed $c^{*}$ is given. To prove the existence of traveling wave solutions, an invariant cone is constructed by upper and lower solutions and Schauder's fixed point theorem is applied. The nonexistence of traveling wave solutions is proved by two-sided Laplace transform. However, to apply two-sided Laplace transform, the prior estimate of exponential decrease of traveling wave solutions is needed. For this aim, a new method is proposed, which can be applied to reactiondiffusion systems consisting of more than three equations.

\section{Introduction}

In the past and at present, cholera has been a serious threat to human health, which is an acute, diarrheal illness caused by infection of the intestine with the bacterium Vibrio cholera. An estimated 3-5 million cases and over 100,000 deaths occur each year around the world [1]. The cholera bacterium is usually found in water or food sources that have been contaminated by feces from a person infected with cholera. Cholera is most likely to be found and spread in places with inadequate water treatment, poor sanitation, and inadequate hygiene. Therefore, cholera outbreaks have been occurring in developing countries-for example, Iraq (20072008), Guinea Bissau (2008), Zimbabwe (2008-2009), Haiti (2010), Democratic Republic of Congo (2011-2012), and Sierra Leone (2012) [2].

Many mathematical models were proposed to understand the propagation mechanism of cholera, the earlier one of which was established by Capasso and Paveri-Fontana [3] to study the 1973 cholera epidemic in the Mediterranean region as follows:

$$
\frac{d I}{d t}=g(B)-a_{22} I
$$

$$
\frac{d B}{d t}=-a_{11} B+a_{12} I
$$

where $B(t)$ and $I(t)$ denote the concentrations of the pathogen and the infective populations, respectively. In addition, Codeço [4] investigated the role of the aquatic pathogen in dynamics of cholera through the following susceptibleinfective-pathogen mode:

$$
\begin{gathered}
\frac{d S}{d t}=n(H-S)-a \frac{S B}{K+B}, \\
\frac{d I}{d t}=a \frac{S B}{K+B}-r I, \\
\frac{d B}{d t}=e I-(m b-n b) B,
\end{gathered}
$$

where $S(t)$ is the susceptible individuals. In this model, human is divided into two groups: the susceptible and the infective. As pointed out in [4-8], bacterium Vibrio cholera can spread by direct human-to-human and indirect environment-to-human modes. To understand the complex dynamics of cholera, model (2) is extended by [6, 9-15], and so forth. 
In all previous models, the influences of space distribution of human on the transmission of cholera are omitted. Cholera usually spreads in spacial wave [16]. Cholera bacteria live in rivers and interact with the plankton on the surface of the water [17]. When individuals drink contaminated water and are infected, then they will release cholera bacteria through excretion [18]. Capasso et al. [19-22] developed model (1) by incorporating the bacterium diffusion in a bounded area and studied the existence and stability of solutions. To deeply investigate the interaction of transmission modes and bacterium diffusion, Bertuzzo et al. [23, 24] incorporated patchy structure into model (2) and supposed that the pathogen in water could diffuse among these patches. Furthermore, Mari et al. [25] studied the influence of diffusion of both human and pathogen on cholera dynamics through a patchy model.

Infectious case usually is found firstly at some location and then spreads to other areas. Consequently, the most important question for cholera is as follows: what is the spreading speed of cholera? However, the above spacial models mainly focus on the stability of solutions, not the spreading speed. Traveling wave solution is an important tool used to study the spreading speed of infectious diseases [2628]. Based on Capasso's model (1), Zhao and Wang [29], Xu and Zhao [30], Jin and Zhao [31], and Hsu and Yang [32] studied the influences of pathogen diffusion on the spread speed of cholera.

In above diffusive cholera models, diffusion of aquatic pathogen is neglected. In this paper, we investigate the effects of the disease-related death and aquatic pathogen in cholera epidemic dynamics by developing model (2). Based on model (2) and ignoring natural birth and death, a general diffusive epidemic cholera model incorporating the disease-related death and aquatic pathogen dynamics can be formulated as the following reaction-diffusion system:

$$
\begin{gathered}
\frac{\partial S}{\partial t}=-f(B) S, \\
\frac{\partial I}{\partial t}=f(B) S-\delta I, \\
\frac{\partial B}{\partial t}=d \frac{\partial^{2} B}{\partial x^{2}}+\gamma I-m B,
\end{gathered}
$$

where $S=S(x, t)$ and $I=I(x, t)$ denote the concentrations of susceptible and infected individuals, respectively, and $B=$ $B(x, t)$ is the concentration of the infectious agents. $\delta$ is the disease-related death rate, $\gamma$ denotes the contribution of each infected person to the concentration of cholera, and $m$ is the net death rate of the vibrio. $f(B)$ is the environment-tohuman transmission incidence. Similar to [15], we assume that $f(B)$ satisfies

$$
\begin{aligned}
& \text { (A1) } f(0)=0, \lim _{B \rightarrow+\infty} f(B)<+\infty, f^{\prime}(B)>0,-M_{0} \leq \\
& f^{\prime \prime}(B) \leq 0 \text { for } B \geq 0 .
\end{aligned}
$$

From hypothesis (A1), we have $f(B) \leq f^{\prime}(0) B$.

In this paper, we study the traveling wave solutions of model (3). The formula for minimal wave speed $c^{*}$ is given. To prove the existence of traveling wave solutions for $c>c^{*}$, an invariant cone is constructed and Schauder's fixed point theorem is introduced. Schauder's fixed point theorem is applied widely to prove the existence of traveling wave solutions (e.g., $[26,33,34])$. However, unlike Wang and $\mathrm{Wu}[34]$, the cone in our paper is bounded. Motivated by [34-37], we prove the nonexistence of traveling wave solutions for $c<c^{*}$ by two-sided Laplace transform, which was firstly introduced to prove the nonexistence of traveling wave solutions by Carr and Chmaj [37] and then was applied by [34-36]. To apply two-sided Laplace transform, the exponential decrease of traveling wave solutions is needed, which is proved in [34] by analysis method. However, it cannot be applied to our model due to the nonlinearity of cholera incidence. Therefore, in this paper, a new method is proposed to get the exponential decrease of traveling wave solutions, which is inspired by the proof of Stable Manifold Theorem in [38]. In addition, our method can be applied to reaction-diffusion systems consisting of more than three equations.

This paper is organized as follows. Section 2 is focused on the existence of traveling wave solutions. Firstly, the existence of traveling wave solutions for original system is proved to be equivalent to that of a new simple system. Then, two pairs of upper and lower solutions are constructed to get an invariant cone and Schauder's fixed point theorem is applied for new system. Section 3 is devoted to the nonexistence of traveling wave solutions. For this aim, a new method is proposed to show the exponential decrease of traveling wave solutions and two-sided Laplace transform is used.

\section{Existence of Traveling Wave Solutions}

For convenience in discussing the model, we introduce dimensionless variables and parameters. Setting

$$
\begin{gathered}
u_{1}=\frac{m}{\delta} S, \quad u_{2}=I, \quad u_{3}=\frac{m}{\gamma} B, \\
\tau=m t, \quad y=\sqrt{\frac{m}{d}} x,
\end{gathered}
$$

we obtain

$$
\begin{gathered}
u_{1, \tau}=-g\left(u_{3}\right) u_{1}, \\
u_{2, \tau}=\kappa\left[g\left(u_{3}\right) u_{1}-u_{2}\right], \\
u_{3, \tau}=u_{3, y y}+u_{2}-u_{3},
\end{gathered}
$$

where

$$
\begin{gathered}
\kappa=\frac{\delta}{m}, \quad g\left(u_{3}\right)=f \frac{\left(\gamma u_{3} / m\right)}{m}, \\
u_{i, \tau}=\frac{\partial u_{i}}{\partial \tau}, \quad u_{3, y y}=\frac{\partial^{2} u_{3}}{\partial y^{2}} .
\end{gathered}
$$

Obviously, $g\left(u_{3}\right)$ also satisfies assumption (A1) with $M_{0}$ being replaced by a new constant $\bar{M}_{0}$. 
A traveling wave solution of system (5) is a nonnegative nontrivial solution of the form

$$
\begin{gathered}
u_{1}(y, \tau)=U(\xi), \\
u_{2}(y, \tau)=W(\xi), \\
u_{3}(y, \tau)=V(\xi), \quad \xi=y+c \tau
\end{gathered}
$$

satisfying boundary condition

$$
\begin{aligned}
& (U(-\infty), W(-\infty), V(-\infty))=\left(U^{0}, 0,0\right), \\
& (U(+\infty), W(+\infty), V(+\infty))=\left(U^{1}, 0,0\right),
\end{aligned}
$$

where $U^{0}>U^{1} \geq 0$.

Before giving the main theorem, we introduce the equation for minimal wave speed:

$$
\Delta(c)=b_{3} c^{6}+b_{2} c^{4}+b_{1} c^{2}+b_{0}=0,
$$

where

$$
\begin{gathered}
b_{3}=-2 \kappa\left[2 g^{\prime}(0) U^{0}-1\right]-1-\kappa^{2} \\
b_{2}=-2 \kappa\left[9 g^{\prime}(0) U^{0}-4\right]-2 \kappa^{2}-2 \kappa^{3}-6 \kappa^{2} g^{\prime}(0) U^{0}-4, \\
b_{1}=8 \kappa^{2}-8 \kappa^{3}-\kappa^{4}-36 \kappa^{2} g^{\prime}(0) U^{0} \\
+27 \kappa^{2}\left(g^{\prime}(0) U^{0}\right)^{2}+6 \kappa^{3} g^{\prime}(0) U^{0} \\
b_{0}=4 \kappa^{4}\left[g^{\prime}(0) U^{0}-1\right] .
\end{gathered}
$$

The main result of this section is given as follows.

Theorem 1. Suppose $U^{0}>1 / g^{\prime}(0)$. Then, there exists a positive constant $c^{*}$ which is the only positive root of (9). For any $>c^{*}$, system (5) has a traveling wave solution $(U(y+$ $c \tau), W(y+c \tau), V(y+c \tau))$ satisfying boundary condition (8) such that $U(\xi)$ is nonincreasing in $\mathbb{R}$. Furthermore, one has that

$$
\begin{aligned}
\int_{-\infty}^{+\infty} W(\eta) d \eta & =\int_{-\infty}^{+\infty} V(\eta) d \eta=c\left(U^{0}-U^{1}\right) \\
0 & \leq V(\xi) \leq U^{0}-U^{1} .
\end{aligned}
$$

To study the existence of traveling wave solutions, using constant variation method in the second equation of (5) gives

$$
\begin{aligned}
u_{2}(y, \tau) & =\kappa \int_{-\infty}^{\tau} e^{-\kappa(\tau-\zeta)} g\left(u_{3}(y, \zeta)\right) u_{1}(y, \zeta) d \zeta \\
& =\kappa \int_{0}^{+\infty} e^{-\kappa \eta} g\left(u_{3}(y, \tau-\eta)\right) u_{1}(y, \tau-\eta) d \eta
\end{aligned}
$$

Then, system (5) changes into

$$
\begin{gathered}
u_{1, \tau}=-g\left(u_{3}(y, \tau)\right) u_{1}(y, \tau) \\
u_{3, \tau}=u_{3, y y}+\kappa \int_{0}^{+\infty} e^{-\kappa \eta} g\left(u_{3}(y, \tau-\eta)\right) u_{1}(y, \tau-\eta) d \eta \\
-u_{3}(y, \tau)
\end{gathered}
$$

Lemma 2. $(U(y+c \tau), V(y+c \tau))$ is a traveling wave solution of system (13) satisfying boundary condition

$$
\begin{gathered}
U(-\infty)=U^{0}, \quad U(+\infty)=U^{1}, \\
V(-\infty)=V(+\infty)=0,
\end{gathered}
$$

if and only if $(U(y+c \tau), W(y+c \tau), V(y+c \tau))$ is a traveling wave solution of system (5) satisfying boundary condition (8), where

$$
\begin{aligned}
& W(y+c \tau) \\
& \quad=\kappa \int_{0}^{+\infty} e^{-\kappa \eta} g(V(y+c \tau-c \eta)) U(y+c \tau-c \eta) d \eta .
\end{aligned}
$$

Proof. Assume $(U(y+c \tau), V(y+c \tau))$ is a traveling wave solution of system (13) satisfying boundary condition (14). Obviously, $(U(y+c \tau), W(y+c \tau), V(y+c \tau))$ is a solution of system (5). To prove the necessity, it is enough to show that $W(+\infty)=W(-\infty)=0$. Consider

$$
\begin{aligned}
\lim _{\xi \rightarrow \pm \infty} W(\xi) & =\lim _{\xi \rightarrow \pm \infty} \kappa \int_{0}^{+\infty} e^{-\kappa \eta} g(V(\xi-c \eta)) U(\xi-c \eta) d \eta \\
& =\lim _{\xi \rightarrow \pm \infty} \frac{(\kappa / c) \int_{-\infty}^{\xi} e^{\kappa s / c} g(V(s)) U(s) d s}{e^{\kappa \xi / c}} \\
& =\lim _{\xi \rightarrow \pm \infty} g(V(\xi)) U(\xi)=0,
\end{aligned}
$$

where the third equality is due to L'Hopital principal. The sufficiency is clear and is omitted. The proof is completed.

From Lemma 2, we only need to study traveling wave solutions of (13) satisfying boundary condition (14). Substituting traveling profile

$$
u_{1}(y, \tau)=U(\xi), \quad u_{3}(y, \tau)=V(\xi), \quad \xi=y+c \tau
$$

into system (13) yields the following equations:

$$
c U^{\prime}=-g(V(\xi)) U(\xi),
$$

$c V^{\prime}=V^{\prime \prime}+\kappa \int_{0}^{+\infty} e^{-\kappa \eta} g(V(\xi-c \eta)) U(\xi-c \eta) d \eta-V(\xi)$

where / denotes the derivative with respect to $\xi$.

In the following, we will use Schauder's fixed point theorem to prove the existence of traveling wave solutions. To achieve this goal, we firstly linearize the second equation of $(18)$ at $\left(U^{0}, 0\right)$ and obtain

$$
c \phi^{\prime}=\phi^{\prime \prime}+\kappa g^{\prime}(0) U^{0} \int_{0}^{+\infty} e^{-\kappa \eta} \phi(\xi-c \eta) d \eta-\phi(\xi) .
$$

Substituting $\phi(\xi)=e^{\lambda \xi} v$ into (19), we get the characteristic equation

$$
\Theta(\lambda)=\lambda^{2}-c \lambda+\kappa g^{\prime}(0) U^{0} \int_{0}^{+\infty} e^{-(\kappa+c \lambda) \eta} d \eta-1=0
$$


that is,

$$
H(\lambda)=\lambda^{3}+a_{2} \lambda^{2}+a_{1} \lambda+a_{0}=0,
$$

where $a_{0}=\kappa\left[g^{\prime}(0) U^{0}-1\right] / c, a_{1}=-(1+\kappa)<0$, and $a_{2}=$ $\left(\kappa-c^{2}\right) / c$. To investigate distribution of roots of (21), denote

$$
\begin{gathered}
p=a_{1}-\frac{a_{2}^{2}}{3}, \\
q=\frac{2 a_{2}^{3}}{27}-\frac{a_{1} a_{2}}{3}+a_{0}, \\
\Delta_{0}=\frac{q^{2}}{4}+\frac{p^{3}}{27},
\end{gathered}
$$

and introduce the following lemma [39].

Lemma 3. (a) If $\Delta_{0}>0,(21)$ has one real root and two nonreal complex conjugate roots. real.

(b) If $\Delta_{0}=0,(21)$ has a multiple root and all its roots are

(c) If $\Delta_{0}<0$, (21) has three distinct real roots.

Then, we get the following lemma about the distribution of eigenvalues.

Lemma 4. Assume $U^{0}>1 / g^{\prime}(0)$. Then, there exists a constant $c^{*}>0$ which is the only positive root of (9) such that

(a) if $0<c<c^{*}$, (21) has a negative real root and two nonreal complex conjugate roots with positive real parts;

(b) if $c=c^{*},(21)$ has a negative real root and a positive real multiple root;

(c) if $c>c^{*}$, (21) has a negative real root and two different positive real roots;

(d) one can assume $c>c^{*}$ and let $\lambda_{1}<\lambda_{2}$ be the two positive roots of (21); then $H\left(\lambda_{1}+\varepsilon\right)<0$ if $0<\varepsilon<$ $\lambda_{2}-\lambda_{1}$.

Proof. Obviously, $b_{3}<0, b_{2}<0$, and $b_{0}>0$. By Descartes' rule of signs, $\Delta(c)=0$ has only one positive root $c^{*}$ such that $\Delta<0$ for $c>c^{*}$ and $\Delta>0$ for $0<c<c^{*}$. Direct calculations show that $\Delta_{0}=\Delta /\left(108 c^{4}\right)$. Since $a_{0}>0$ and $a_{1}<0$, Descartes' rule of signs shows that (21) has only one negative real root and Routh-Hurwitz criterion indicates that (21) has roots with positive real parts. Then, the combination of Lemma 3 and above analysis completes the proof of (a)-(c). (d) is obviously true, since $H(\lambda)$ is a cubic polynomial.

In this section, we always suppose $U^{0}>1 / g^{\prime}(0)$ and $c>$ $c^{*}$ unless other conditions are specified. Denote $\lambda_{1}<\lambda_{2}$ to be the two positive roots of (21) and define

$$
\begin{gathered}
\underline{U}(\xi)=\max \left\{U^{0}-\sigma e^{\alpha \xi}, 0\right\}, \\
\bar{V}(\xi)=\min \left\{e^{\lambda_{1} \xi}, V^{0}\right\}, \\
\underline{V}(\xi)=\max \left\{e^{\lambda_{1} \xi}\left(1-M e^{\varepsilon \xi}\right), 0\right\},
\end{gathered}
$$

where $V^{0}=J U^{0}, J=\lim _{V \rightarrow+\infty} g(V)$.
Lemma 5. The function $\bar{V}(\xi)$ satisfies inequality

$$
c \bar{V}^{\prime} \geq \bar{V}^{\prime \prime}+\kappa U^{0} \int_{0}^{+\infty} e^{-\kappa \eta} g(\bar{V}(\xi-c \eta)) d \eta-\bar{V}(\xi)
$$

for any $\xi \neq \ln V^{0} / \lambda_{1}$.

Proof. Firstly, assume $\xi<\ln V^{0} / \lambda_{1}$ and, therefore, $\bar{V}(\xi)=$ $e^{\lambda_{1} \xi}$. Since $\bar{V}(\xi)$ satisfies (19) and $g^{\prime \prime}(V) \leq 0$ for any $V \geq 0$, we have

$$
\begin{aligned}
c \bar{V}^{\prime}-\bar{V}^{\prime \prime}-\kappa U^{0} \int_{0}^{+\infty} e^{-\kappa \eta} g(\bar{V}(\xi-c \eta)) d \eta+\bar{V}(\xi) \\
=c \bar{V}^{\prime}-\bar{V}^{\prime \prime}-\kappa g^{\prime}(0) U^{0} \int_{0}^{+\infty} e^{-\kappa \eta} \bar{V}(\xi-c \eta) d \eta+\bar{V}(\xi) \\
\quad-\frac{\kappa U^{0}}{2} \int_{0}^{+\infty} e^{-\kappa \eta}\left[g^{\prime \prime}(\theta \bar{V}(\xi-c \eta))\right][\bar{V}(\xi-c \eta)]^{2} d \eta \\
=-\frac{\kappa U^{0}}{2} \int_{0}^{+\infty} e^{-\kappa \eta}\left[g^{\prime \prime}(\theta \bar{V}(\xi-c \eta))\right][\bar{V}(\xi-c \eta)]^{2} d \eta \geq 0,
\end{aligned}
$$

where $0<\theta<1$.

Secondly, set $\xi>\ln V^{0} / \lambda_{1}$, which implies $\bar{V}(\xi)=V^{0}$. We have that

$$
\begin{aligned}
c \bar{V}^{\prime}- & \bar{V}^{\prime \prime}-\kappa U^{0} \int_{0}^{+\infty} e^{-\kappa \eta} g(\bar{V}(\xi-c \eta)) d \eta+\bar{V}(\xi) \\
& \geq V^{0}-\kappa g\left(V^{0}\right) U^{0} \int_{0}^{+\infty} e^{-\kappa \eta} d \eta \\
& =V^{0}-g\left(V^{0}\right) U^{0} \\
& =\left[J-g\left(V^{0}\right)\right] U^{0} \\
& \geq 0 .
\end{aligned}
$$

The proof is completed.

Lemma 6. For $\alpha>0$ sufficiently small and $\sigma>0$ sufficiently large, the function $\underline{U}(\xi)$ satisfies

$$
c \underline{U^{\prime}} \leq-g(\bar{V}(\xi)) \underline{U}(\xi)
$$

for any $\xi \neq 1 / \alpha \ln \left(U^{0} / \sigma\right)$.

Proof. Let $\sigma>0$ be sufficiently large to ensure $1 / \alpha \ln \left(U^{0} / \sigma\right)<$ $\ln V^{0} / \lambda_{1}$. When $\xi>1 / \alpha \ln \left(U^{0} / \sigma\right)$, then $\underline{U}(\xi)=0$ and the 
lemma is obviously true. Now, suppose $\xi<1 / \alpha \ln \left(U^{0} / \sigma\right)$. Then, $\underline{U}(\xi)=U^{0}-\sigma e^{\alpha \xi}$ and

$$
\begin{aligned}
&-c \underline{U}^{\prime}-g(\bar{V}(\xi)) \underline{U}(\xi) \\
&=c \sigma \alpha e^{\alpha \xi}-\left[g^{\prime}(0) \bar{V}(\xi)+\frac{g^{\prime \prime}(\theta \bar{V}(\xi))}{2}(\bar{V}(\xi))^{2}\right] \\
& \times\left(U^{0}-\sigma e^{\alpha \xi}\right) \\
&= c \sigma \alpha e^{\alpha \xi}-g^{\prime}(0) U^{0} e^{\lambda_{1} \xi}+g^{\prime}(0) \bar{V}(\xi) \sigma e^{\alpha \xi} \\
&-\frac{g^{\prime \prime}(\theta \bar{V}(\xi))}{2}(\bar{V}(\xi))^{2}\left(U^{0}-\sigma e^{\alpha \xi}\right) \\
& \geq e^{\alpha \xi}\left[c \sigma \alpha-g^{\prime}(0) U^{0} e^{\left(\lambda_{1}-\alpha\right)(1 / \alpha) \ln \left(U^{0} / \sigma\right)}\right] \\
&= e^{\alpha \xi}\left[c \sigma \alpha-g^{\prime}(0) U^{0} e^{\left(\lambda_{1}-\alpha\right)}\left(\frac{U^{0}}{\sigma}\right)^{1 / \alpha}\right],
\end{aligned}
$$

where $0<\theta<1$. Let $\sigma=1 / \alpha$. Since

$$
\lim _{\alpha \rightarrow 0^{+}}\left(\frac{U^{0}}{\sigma}\right)^{1 / \alpha}=\lim _{\alpha \rightarrow 0^{+}}\left(U^{0} \alpha\right)^{1 / \alpha}=0,
$$

we can find $\alpha>0$ sufficiently small and $\sigma>0$ sufficiently large such that

$$
-c \underline{U}^{\prime}-g(\bar{V}(\xi)) \underline{U}(\xi) \geq 0 .
$$

Thus, the proof is completed.

Lemma 7. Let $\varepsilon=\min \left\{\alpha, \lambda_{1}, \lambda_{2}-\lambda_{1}\right\} / 2$. Then, for $M>0$ large enough, the function $\underline{V}(\xi)$ satisfies

$$
c \underline{V}^{\prime} \leq \underline{V}^{\prime \prime}+\kappa \int_{0}^{+\infty} e^{-\kappa \eta} g(\underline{V}(\xi-c \eta)) \underline{U}(\xi-c \eta) d \eta-\underline{V}(\xi)
$$

for any $\xi \neq 1 / \varepsilon \ln (1 / M)$.

Proof. It is clear that $\underline{U}(\xi)=0$ if and only if $\xi=1 / \alpha \ln \left(U^{0} / \sigma\right)$, that $\underline{V}(\xi)=0$ if and only if $\xi=1 / \varepsilon \ln (1 / M)$, and that $1 / \varepsilon \ln (1 / M)<1 / \alpha \ln \left(U^{0} / \sigma\right)$ if and only if $M>\left(\sigma / U^{0}\right)^{(\varepsilon / \alpha)}$. Let $M>\left(\sigma / U^{0}\right)^{(\varepsilon / \alpha)}$. When $\xi>1 / \varepsilon \ln (1 / M)$, then $e^{\lambda_{1} \xi}(1-$ $\left.M e^{\varepsilon \xi}\right)<0, \underline{V}(\xi)=0$, and Lemma 7 holds.
In this paragraph, assume $\xi<1 / \varepsilon \ln (1 / M)$. Then, $\xi<$ $1 / \alpha \ln \left(U^{0} / \sigma\right), \underline{U}(\xi)=U^{0}-\sigma e^{\alpha \xi}>0$, and $\underline{V}(\xi)=e^{\lambda_{1} \xi}(1-$ $\left.M e^{\varepsilon \xi}\right)>0$. To prove this lemma, it is enough to show

$$
\begin{aligned}
& 0 \leq e^{-\lambda_{1} \xi}\left[\underline{V}^{\prime \prime}-c \underline{V}^{\prime}+\kappa \int_{0}^{+\infty} e^{-\kappa \eta} g(\underline{V}(\xi-c \eta))\right. \\
& \times \underline{U}(\xi-c \eta) d \eta-\underline{V}(\xi)] \\
& =\lambda_{1}^{2}-M\left(\lambda_{1}+\varepsilon\right)^{2} e^{\varepsilon \xi}-c \lambda_{1}+c M\left(\lambda_{1}+\varepsilon\right) e^{\varepsilon \xi}-1 \\
& +M e^{\varepsilon \xi}+e^{-\lambda_{1} \xi} \kappa \int_{0}^{+\infty} e^{-\kappa \eta} \\
& \times\left[g^{\prime}(0) \underline{V}(\xi-c \eta)\right. \\
& \left.+\frac{g^{\prime \prime}(\theta \underline{V}(\xi-c \eta))}{2} \underline{V}^{2}(\xi-c \eta)\right] \\
& \times \underline{U}(\xi-c \eta) d \eta \\
& =\lambda_{1}^{2}-c \lambda_{1}+\kappa g^{\prime}(0) U^{0} \int_{0}^{+\infty} e^{-\left(\kappa+c \lambda_{1}\right) \eta} d \eta-1 \\
& +M\left[-\left(\lambda_{1}+\varepsilon\right)^{2}+c\left(\lambda_{1}+\varepsilon\right)\right. \\
& \left.-\kappa g^{\prime}(0) U^{0} \int_{0}^{+\infty} e^{-\left(\kappa+c\left(\lambda_{1}+\varepsilon\right)\right) \eta} d \eta+1\right] e^{\varepsilon \xi} \\
& -\kappa g^{\prime}(0) \sigma e^{\alpha \xi} \int_{0}^{+\infty} e^{-\left(\kappa+c \lambda_{1}+c \alpha\right) \eta} d \eta \\
& +M \kappa g^{\prime}(0) \sigma e^{(\alpha+\varepsilon) \xi} \int_{0}^{+\infty} e^{-\left(\kappa+c \lambda_{1}+c \varepsilon+c \alpha\right) \eta} d \eta \\
& +\frac{\kappa}{2} e^{\lambda_{1} \xi} \int_{0}^{+\infty} e^{-\left(\kappa+2 c \lambda_{1}\right) \eta} g^{\prime \prime}(\theta \underline{V}(\xi-c \eta)) \\
& \times\left(1-M e^{\varepsilon \xi} e^{-\varepsilon c \eta}\right)^{2} \underline{U}(\xi-c \eta) d \eta \\
& =M \frac{-c H\left(\lambda_{1}+\varepsilon\right)}{\kappa+c \lambda_{1}+c \varepsilon} e^{\varepsilon \xi}-\frac{\kappa g^{\prime}(0) \sigma}{\kappa+c \lambda_{1}+c \alpha} e^{\alpha \xi} \\
& +\frac{M \kappa g^{\prime}(0) \sigma}{\kappa+c \lambda_{1}+c \varepsilon+c \alpha} e^{(\alpha+\varepsilon) \xi} \\
& +\frac{\kappa}{2} e^{\lambda_{1} \xi} \int_{0}^{+\infty} e^{-\left(\kappa+2 c \lambda_{1}\right) \eta} g^{\prime \prime}(\theta \underline{V}(\xi-c \eta)) \\
& \times\left(1-M e^{\varepsilon \xi} e^{-\varepsilon c \eta}\right)^{2} \underline{U}(\xi-c \eta) d \eta,
\end{aligned}
$$

where $0<\theta<1$. Since

$$
\begin{aligned}
0 \geq \frac{\kappa}{2} e^{\lambda_{1} \xi} \int_{0}^{+\infty} e^{-\left(\kappa+2 c \lambda_{1}\right) \eta} g^{\prime \prime}(\theta \underline{V}(\xi-c \eta)) \\
\times\left(1-M e^{\varepsilon \xi} e^{-\varepsilon c \eta}\right)^{2} \underline{U}(\xi-c \eta) d \eta
\end{aligned}
$$




$$
\begin{aligned}
& \geq-\frac{\kappa}{2} e^{\lambda_{1} \xi} \int_{0}^{+\infty} e^{-\left(\kappa+2 c \lambda_{1}\right) \eta} \bar{M}_{0} U^{0} d \eta \\
& =-\frac{\kappa \bar{M}_{0} U^{0}}{2\left(\kappa+2 c \lambda_{1}\right)} e^{\lambda_{1} \xi},
\end{aligned}
$$

we only need to show

$$
\begin{aligned}
M \frac{-c H\left(\lambda_{1}+\varepsilon\right)}{\kappa+c \lambda_{1}} \geq & \frac{\kappa g^{\prime}(0) \sigma}{\kappa+c \lambda_{1}+c \alpha} e^{(\alpha-\varepsilon) \xi} \\
& +\frac{\kappa \bar{M}_{0} U^{0}}{2\left(\kappa+2 c \lambda_{1}\right)} e^{\left(\lambda_{1}-\varepsilon\right) \xi}
\end{aligned}
$$

Since $\xi<1 / \alpha \ln \left(U^{0} / \sigma\right)$, then

$$
\begin{aligned}
e^{(\alpha-\varepsilon) \xi} & <\left(U^{0} \sigma^{-1}\right)^{(\alpha-\varepsilon) / \alpha}, \\
e^{\left(\lambda_{1}-\varepsilon\right) \xi} & <\left(U^{0} \sigma^{-1}\right)^{\left(\lambda_{1}-\varepsilon\right) / \alpha} .
\end{aligned}
$$

Since $H\left(\lambda_{1}+\varepsilon\right)<0$, inequality (34) satisfies if

$$
\begin{aligned}
M>- & {\left[\frac{\kappa g^{\prime}(0) \sigma}{\kappa+c \lambda_{1}+c \alpha}\left(U^{0} \sigma^{-1}\right)^{(\alpha-\varepsilon) / \alpha}\right.} \\
& \left.+\frac{\kappa \bar{M}_{0} U^{0}}{2\left(\kappa+2 c \lambda_{1}\right)}\left(U^{0} \sigma^{-1}\right)^{\left(\lambda_{1}-\varepsilon\right) / \alpha}\right] \\
& \times \frac{\kappa+c \lambda_{1}}{c H\left(\lambda_{1}+\varepsilon\right)} .
\end{aligned}
$$

The proof is completed.

To apply Schauder's fixed point theorem, we will introduce a topology in $C\left(\mathbb{R}, \mathbb{R}^{2}\right)$. Let $\mu$ be a positive constant which will be specified in the following. For $\Phi(\xi)=$ $\left(\phi_{1}(\xi), \phi_{2}(\xi)\right)$, define

$$
\begin{gathered}
|\Phi(\cdot)|_{\mu}=\max \left\{\sup _{\xi \in \mathbb{R}}\left|\phi_{1}(\xi)\right| e^{-\mu|\xi|}, \sup _{\xi \in \mathbb{R}}\left|\phi_{2}(\xi)\right| e^{-\mu|\xi|}\right\}, \\
B_{\mu}\left(\mathbb{R}, \mathbb{R}^{2}\right)=\left\{\Phi(\cdot) \in C\left(\mathbb{R}, \mathbb{R}^{2}\right):|\Phi(\cdot)|_{\mu}<+\infty\right\} .
\end{gathered}
$$

We will find traveling wave solutions in the following profile set:

$$
\begin{gathered}
\Gamma=\left\{(U(\cdot), V(\cdot)) \in C\left(\mathbb{R}, \mathbb{R}^{2}\right): \underline{U}(\xi) \leq U(\xi) \leq U^{0},\right. \\
\underline{V}(\xi) \leq V(\xi) \leq \bar{V}(\xi) \text { for any } \xi \in \mathbb{R}\} .
\end{gathered}
$$

Obviously, $\Gamma$ is closed and convex in $C\left(\mathbb{R}, \mathbb{R}^{2}\right)$. Firstly, we change system (18) into the following form:

$$
\begin{gathered}
c U^{\prime}+\beta_{1} U(\xi)=H_{1}(U, V)(\xi), \\
-V^{\prime \prime}+c V^{\prime}+V(\xi)=H_{2}(U, V)(\xi),
\end{gathered}
$$

where $\beta_{1}>\lim _{V \rightarrow+\infty} g(V)$,

$$
\begin{gathered}
H_{1}(U, V)(\xi)=\left[\beta_{1}-g(V(\xi))\right] U(\xi), \\
H_{2}(U, V)(\xi)=\kappa \int_{0}^{+\infty} e^{-\kappa \eta} g(V(\xi-c \eta)) U(\xi-c \eta) d \eta .
\end{gathered}
$$

Suppose $\Lambda_{1}<0<\Lambda_{2}$ are the two roots of equation $\Lambda^{2}-c \Lambda-$ $1=0$. Furthermore, define $F=\left(F_{1}, F_{2}\right): \Gamma \rightarrow C\left(\mathbb{R}, \mathbb{R}^{2}\right)$ by

$$
\begin{aligned}
F_{1}(U(\cdot), V(\cdot))(\xi) & =\frac{1}{c} \int_{-\infty}^{\xi} e^{-\left(\beta_{1} / c\right)(\xi-t)} H_{1}(U, V)(t) d t \\
F_{2}(U(\cdot), V(\cdot))(\xi)= & \frac{1}{\Lambda_{2}-\Lambda_{1}} \\
& \times\left[\int_{-\infty}^{\xi} e^{\Lambda_{1}(\xi-t)} H_{2}(U, V)(t) d t\right. \\
& \left.\quad+\int_{\xi}^{+\infty} e^{\Lambda_{2}(\xi-t)} H_{2}(U, V)(t) d t\right] .
\end{aligned}
$$

In the remainder of this paper, it is always assumed that $\mu<$ $\min \left\{\beta_{1} / c, \kappa / c,-\Lambda_{1}, \Lambda_{2}\right\}$.

Lemma 8. Consider $F(\Gamma) \subset \Gamma$.

Proof. Let $(U(\cdot), V(\cdot)) \in \Gamma$; that is $\underline{U}(\xi) \leq U(\xi) \leq U^{0}, \underline{V}(\xi) \leq$ $V(\xi) \leq \bar{V}(\xi)$. Then, we need to prove that

$$
\begin{gathered}
\underline{U}(\xi) \leq F_{1}(U(\cdot), V(\cdot))(\xi) \leq U^{0}, \\
\underline{V}(\xi) \leq F_{2}(U(\cdot), V(\cdot))(\xi) \leq \bar{V}(\xi) .
\end{gathered}
$$

First of all, we have

$$
\begin{aligned}
F_{1}(U(\cdot), V(\cdot))(\xi) & =\frac{1}{c} \int_{-\infty}^{\xi} e^{-\left(\beta_{1} / c\right)(\xi-t)} H_{1}(U, V)(t) d t \\
& \leq \frac{1}{c} \int_{-\infty}^{\xi} e^{-\left(\beta_{1} / c\right)(\xi-t)} \beta_{1} U^{0} d t \\
& =U^{0} .
\end{aligned}
$$

From Lemma 6 and system (39), we get

$$
\begin{aligned}
c \underline{U^{\prime}}+\beta_{1} \underline{U}(\xi) & \leq H_{1}(\underline{U}, \bar{V})(\xi) \\
& =\left[\beta_{1}-g(\bar{V}(\xi))\right] \underline{U}(\xi) \\
& \leq\left[\beta_{1}-g(V(\xi))\right] U(\xi) \\
& =H_{1}(U, V)(\xi),
\end{aligned}
$$


where the second inequality is due to $\beta_{1}>\lim _{V \rightarrow+\infty} g(V)$. Then,

$$
\begin{aligned}
F_{1}(U(\cdot), V(\cdot))(\xi) & =\frac{1}{c} \int_{-\infty}^{\xi} e^{-\left(\beta_{1} / c\right)(\xi-t)} H_{1}(U, V)(t) d t \\
& \geq \frac{1}{c} \int_{-\infty}^{\xi} e^{-\left(\beta_{1} / c\right)(\xi-t)}\left[c \underline{U}^{\prime}(t)+\beta_{1} \underline{U}(t)\right] d t \\
& =\underline{U}(\xi) .
\end{aligned}
$$

Therefore, we have proved $\underline{U}(\xi) \leq F_{1}(U(\cdot), V(\cdot))(\xi) \leq U^{0}$.

Now, we study $F_{2}(U(\cdot), V(\cdot))(\xi)$. If $\xi \geq \xi_{0} \triangleq 1 / \varepsilon \ln (1 / M)$, then $\underline{V}(\xi)=0$, which implies that $F_{2}(U(\cdot), V(\cdot))(\xi) \geq \underline{V}(\xi)$ since $\bar{U}(\xi) \geq \underline{U}(\xi) \geq 0, V(\xi) \geq \underline{V}(\xi) \geq 0$. Assume $\xi<\xi_{0}$. From Lemma 7 and system (39), it is clear that

$$
\begin{aligned}
-\underline{V^{\prime \prime}}+c \underline{V^{\prime}}+\underline{V}(\xi) & \leq H_{2}(\underline{U}, \underline{V})(\xi) \\
& =\kappa \int_{0}^{+\infty} e^{-\kappa \eta} g(\underline{V}(\xi-c \eta)) \underline{U}(\xi-c \eta) d \eta \\
& \leq \kappa \int_{0}^{+\infty} e^{-\kappa \eta} g(V(\xi-c \eta)) U(\xi-c \eta) d \eta \\
& =H_{2}(U, V)(\xi)
\end{aligned}
$$

which implies

$$
\begin{aligned}
F_{2}(U(\cdot), V(\cdot))(\xi) & \frac{1}{\Lambda_{2}-\Lambda_{1}}\left[\int_{-\infty}^{\xi} e^{\Lambda_{1}(\xi-t)} H_{2}(U, V)(t) d t\right. \\
& \left.\quad+\int_{\xi}^{+\infty} e^{\Lambda_{2}(\xi-t)} H_{2}(U, V)(t) d t\right] \\
\geq & \frac{1}{\Lambda_{2}-\Lambda_{1}} \int_{-\infty}^{\xi} e^{\Lambda_{1}(\xi-t)}\left[-\underline{V}^{\prime \prime}(t)+c \underline{V^{\prime}}(t)+\underline{V}(t)\right] d t \\
& +\frac{1}{\Lambda_{2}-\Lambda_{1}} \int_{\xi}^{\xi_{0}} e^{\Lambda_{2}(\xi-t)}\left[-\underline{V}^{\prime \prime}(t)+c \underline{V^{\prime}}(t)+\underline{V}(t)\right] d t \\
& +\frac{1}{\Lambda_{2}-\Lambda_{1}} \int_{\xi_{0}}^{+\infty} e^{\Lambda_{2}(\xi-t)}\left[-\underline{V}^{\prime \prime}(t)+c \underline{V^{\prime}}(t)+\underline{V}(t)\right] d t \\
= & \underline{V}(\xi)+\frac{1}{\Lambda_{2}-\Lambda_{1}} e^{\Lambda_{2}\left(\xi-\xi_{0}\right)}\left[\underline{V}^{\prime}\left(\xi_{0}+0\right)-\underline{V^{\prime}}\left(\xi_{0}-0\right)\right] \\
\geq & \underline{V}(\xi),
\end{aligned}
$$

where the final inequality is due to $\underline{V}^{\prime}\left(\xi_{0}+0\right)=0$ and $\underline{V}^{\prime}\left(\xi_{0}-\right.$ $0)<0$. In conclusion, $F_{2}(U(\cdot), V(\cdot)) \overline{(\xi)} \geq \underline{V}(\xi)$ for any $\bar{\xi} \in \mathbb{R}$.

Similarly, we can show $F_{2}(U(\cdot), V(\cdot))(\xi) \leq \bar{V}(\xi)$ and the proof is completed.

Lemma 9. Map $F=\left(F_{1}, F_{2}\right): \Gamma \rightarrow C\left(\mathbb{R}, \mathbb{R}^{2}\right)$ is continuous with respect to the norm $|\cdot|_{\mu}$ in $B_{\mu}\left(\mathbb{R}, \mathbb{R}^{2}\right)$.
Proof. For $\Phi_{1}(\cdot)=\left(U_{1}(\cdot), V_{1}(\cdot)\right), \Phi_{2}(\cdot)=\left(U_{2}(\cdot), V_{2}(\cdot)\right) \in \Gamma$, we have

$$
\begin{aligned}
\mid & H_{1}\left(U_{1}, V_{1}\right)(\xi)-H_{1}\left(U_{2}, V_{2}\right)(\xi) \mid e^{-\mu|\xi|} \\
\leq & \beta_{1}\left|U_{1}(\xi)-U_{2}(\xi)\right| e^{-\mu|\xi|} \\
& +\left|g\left(V_{1}(\xi)\right) U_{1}(\xi)-g\left(V_{2}(\xi)\right) U_{2}(\xi)\right| e^{-\mu|\xi|} \\
\leq & \beta_{1}\left|U_{1}(\cdot)-U_{2}(\cdot)\right|_{\mu} \\
& +\mid g\left(V_{1}(\xi)\right)\left[U_{1}(\xi)-U_{2}(\xi)\right] \\
& \quad+U_{2}(\xi)\left[g\left(V_{1}(\xi)\right)-g\left(V_{2}(\xi)\right)\right] \mid e^{-\mu|\xi|} \\
\leq & \beta_{1}\left|U_{1}(\cdot)-U_{2}(\cdot)\right|_{\mu}+J\left|U_{1}(\xi)-U_{2}(\xi)\right| e^{-\mu|\xi|} \\
& +U^{0} g^{\prime}\left(V^{*}\right)\left|V_{1}(\xi)-V_{2}(\xi)\right| e^{-\mu|\xi|} \\
\leq & \left(\beta_{1}+J\right)\left|U_{1}(\cdot)-U_{2}(\cdot)\right|_{\mu}+U^{0} g^{\prime}(0)\left|V_{1}(\cdot)-V_{2}(\cdot)\right|_{\mu} \\
\leq & M_{1}\left|\Phi_{1}(\cdot)-\Phi_{2}(\cdot)\right|_{\mu},
\end{aligned}
$$

where $M_{1}=\beta_{1}+J+U^{0} g^{\prime}(0)$, and $V^{*}$ is between $V_{1}(\xi)$ and $V_{2}(\xi)$. Therefore, we have

$$
\left|H_{1}\left(\Phi_{1}(\cdot)\right)-H_{1}\left(\Phi_{2}(\cdot)\right)\right|_{\mu} \leq M_{1}\left|\Phi_{1}(\cdot)-\Phi_{2}(\cdot)\right|_{\mu} .
$$

Then,

$$
\begin{aligned}
& \left|F_{1}\left(U_{1}(\cdot), V_{1}(\cdot)\right)(\xi)-F_{1}\left(U_{2}(\cdot), V_{2}(\cdot)\right)(\xi)\right| e^{-\mu|\xi|} \\
& \leq \frac{1}{c} e^{-\mu|\xi|} \int_{-\infty}^{\xi} e^{-\left(\beta_{1} / c\right)(\xi-t)} \\
& \times\left|H_{1}\left(U_{1}, V_{1}\right)(t)-H_{1}\left(U_{2}, V_{2}\right)(t)\right| d t \\
& =\frac{1}{c} e^{-\mu|\xi|-\left(\beta_{1} / c\right) \xi} \int_{-\infty}^{\xi} e^{\left(\beta_{1} / c\right) t+\mu|t|} \\
& \times\left|H_{1}\left(U_{1}, V_{1}\right)(t)-H_{1}\left(U_{2}, V_{2}\right)(t)\right| e^{-\mu|t|} d t \\
& \leq M_{1}\left|\Phi_{1}(\cdot)-\Phi_{2}(\cdot)\right|_{\mu} \frac{1}{c} e^{-\mu|\xi|-\left(\beta_{1} / c\right) \xi} \int_{-\infty}^{\xi} e^{\left(\beta_{1} / c\right) t+\mu|t|} d t .
\end{aligned}
$$

Thus, when $\xi \leq 0$, we get

$$
\begin{aligned}
& \left|F_{1}\left(U_{1}(\cdot), V_{1}(\cdot)\right)(\xi)-F_{1}\left(U_{2}(\cdot), V_{2}(\cdot)\right)(\xi)\right| e^{-\mu|\xi|} \\
& \quad \leq M_{1}\left|\Phi_{1}(\xi)-\Phi_{2}(\xi)\right|_{\mu} \frac{1}{c} e^{\left(\mu-\beta_{1} / c\right) \xi} \int_{-\infty}^{\xi} e^{\left(\beta_{1} / c-\mu\right) t} d t \\
& \quad=\frac{M_{1}}{\beta_{1}-c \mu}\left|\Phi_{1}(\cdot)-\Phi_{2}(\cdot)\right|_{\mu} .
\end{aligned}
$$


When $\xi>0$, it follows that

$$
\begin{aligned}
\mid F_{1} & \left(U_{1}(\cdot), V_{1}(\cdot)\right)(\xi)-F_{1}\left(U_{2}(\cdot), V_{2}(\cdot)\right)(\xi) \mid e^{-\mu|\xi|} \\
\leq & M_{1}\left|\Phi_{1}(\cdot)-\Phi_{2}(\cdot)\right|_{\mu} \frac{1}{c} e^{-\mu|\xi|-\left(\beta_{1} / c\right) \xi} \int_{-\infty}^{\xi} e^{\left(\beta_{1} / c\right) t+\mu|t|} d t \\
= & M_{1}\left|\Phi_{1}(\cdot)-\Phi_{2}(\cdot)\right|_{\mu} \frac{1}{c} e^{-\mu \xi-\left(\beta_{1} / c\right) \xi} \\
& \times\left[\int_{-\infty}^{0} e^{\left(\beta_{1} / c\right) t-\mu t} d t+\int_{0}^{\xi} e^{\left(\beta_{1} / c\right) t+\mu t} d t\right] \\
= & M_{1}\left|\Phi_{1}(\cdot)-\Phi_{2}(\cdot)\right|_{\mu} \frac{1}{c} e^{-\mu \xi-\left(\beta_{1} / c\right) \xi} \\
& \times\left[\frac{2 c^{2} \mu}{\beta_{1}^{2}-c^{2} \mu^{2}}+\frac{c}{\beta_{1}+c \mu} e^{\left(\beta_{1} / c+\mu\right) \xi}\right] \\
\leq & M_{1}\left(\frac{2 c \mu}{\beta_{1}^{2}-c^{2} \mu^{2}}+\frac{1}{\beta_{1}+c \mu}\right)\left|\Phi_{1}(\cdot)-\Phi_{2}(\cdot)\right|_{\mu} \cdot
\end{aligned}
$$

Consequently, we have proved that

$$
\begin{aligned}
& \left|F_{1}\left(U_{1}(\cdot), V_{1}(\cdot)\right)(\xi)-F_{1}\left(U_{2}(\cdot), V_{2}(\cdot)\right)(\xi)\right| e^{-\mu|\xi|} \\
& \quad \leq M_{2}\left|\Phi_{1}(\cdot)-\Phi_{2}(\cdot)\right|_{\mu}
\end{aligned}
$$

for any $\xi \in \mathbb{R}$, where

$$
M_{2}=\max \left\{\frac{M_{1}}{\beta_{1}-c \mu}, M_{1}\left(\frac{2 c \mu}{\beta_{1}^{2}-c^{2} \mu^{2}}+\frac{1}{\beta_{1}+c \mu}\right)\right\} .
$$

That is,

$$
\left|F_{1}\left(\Phi_{1}(\cdot)\right)-F_{1}\left(\Phi_{2}(\cdot)\right)\right|_{\mu} \leq M_{2}\left|\Phi_{1}(\cdot)-\Phi_{2}(\cdot)\right|_{\mu}
$$

In conclusion, $F_{1}: \Gamma \rightarrow C(\mathbb{R}, \mathbb{R})$ is continuous with respect to the norm $|\cdot|_{\mu}$ in $B_{\mu}(\mathbb{R}, \mathbb{R})$.

In addition, consider $F_{2}(U(\xi), V(\xi))$. Firstly, we have

$$
\begin{aligned}
& \left|H_{2}\left(U_{1}, V_{1}\right)(\xi)-H_{2}\left(U_{2}, V_{2}\right)(\xi)\right| e^{-\mu|\xi|} \\
& \leq \kappa e^{-\mu|\xi|} \int_{0}^{+\infty} e^{-\kappa \eta} \mid g\left(V_{1}(\xi-c \eta)\right) U_{1}(\xi-c \eta) \\
& -g\left(V_{2}(\xi-c \eta)\right) U_{2}(\xi-c \eta) \mid d \eta \\
& \leq \kappa e^{-\mu|\xi|} \int_{0}^{+\infty} e^{-\kappa \eta}\left[J\left|U_{1}(\xi-c \eta)-U_{2}(\xi-c \eta)\right|\right. \\
& \quad+U^{0} g^{\prime}(0) \\
& \left.\times\left|V_{1}(\xi-c \eta)-V_{2}(\xi-c \eta)\right|\right] d \eta \\
& \leq\left[J\left|U_{1}(\cdot)-U_{2}(\cdot)\right|_{\mu}+U^{0} g^{\prime}(0)\left|V_{1}(\xi)-V_{2}(\xi)\right|_{\mu}\right] \\
& \quad \times \kappa e^{-\mu|\xi|} \int_{0}^{+\infty} e^{-\kappa \eta+\mu|\xi-c \eta|} d \eta
\end{aligned}
$$

$$
\begin{aligned}
\leq & \left|\Phi_{1}(\cdot)-\Phi_{2}(\cdot)\right|_{\mu} \\
& \times\left[\left(J+U^{0} g^{\prime}(0)\right) \kappa \int_{0}^{+\infty} e^{-\mu|\xi|-\kappa \eta+\mu|\xi-c \eta|} d \eta\right] \\
\leq & \left|\Phi_{1}(\cdot)-\Phi_{2}(\cdot)\right|_{\mu} \\
& \times\left[\left(J+U^{0} g^{\prime}(0)\right) \kappa \int_{0}^{+\infty} e^{-(\kappa-c \mu) \eta} d \eta\right] \\
= & M_{3}\left|\Phi_{1}(\cdot)-\Phi_{2}(\cdot)\right|_{\mu},
\end{aligned}
$$

where $M_{3}=\left(J+U^{0} g^{\prime}(0)\right) \kappa /(\kappa-c \mu)$. Therefore,

$$
\begin{aligned}
& \left|F_{2}\left(U_{1}(\cdot), V_{1}(\cdot)\right)(\xi)-F_{2}\left(U_{2}(\cdot), V_{2}(\cdot)\right)(\xi)\right| e^{-\mu|\xi|} \\
& \leq \frac{e^{-\mu|\xi|}}{\Lambda_{2}-\Lambda_{1}} \\
& \quad \times\left[\int_{-\infty}^{\xi} e^{\Lambda_{1}(\xi-t)}\left|H_{2}\left(U_{1}, V_{1}\right)(t)-H_{2}\left(U_{2}, V_{2}\right)(t)\right| d t\right. \\
& \left.\quad+\int_{\xi}^{+\infty} e^{\Lambda_{2}(\xi-t)}\left|H_{2}\left(U_{1}, V_{1}\right)(t)-H_{2}\left(U_{2}, V_{2}\right)(t)\right| d t\right] \\
& \leq \frac{M_{3} e^{-\mu|\xi|}}{\Lambda_{2}-\Lambda_{1}} \\
& \quad \times\left[\int_{-\infty}^{\xi} e^{\Lambda_{1}(\xi-t)+\mu|t|} d t+\int_{\xi}^{+\infty} e^{\Lambda_{2}(\xi-t)+\mu|t|} d t\right] \\
& \quad \times\left|\Phi_{1}(\cdot)-\Phi_{2}(\cdot)\right|_{\mu} \cdot
\end{aligned}
$$

If $\xi<0$, it holds that

$$
\begin{aligned}
& \left|F_{2}\left(U_{1}(\cdot), V_{1}(\cdot)\right)(\xi)-F_{2}\left(U_{2}(\cdot), V_{2}(\cdot)\right)(\xi)\right| e^{-\mu|\xi|} \\
& \leq \frac{M_{3} e^{\mu \xi}}{\Lambda_{2}-\Lambda_{1}} \\
& \quad \times\left[e^{\Lambda_{1} \xi} \int_{-\infty}^{\xi} e^{-\left(\Lambda_{1}+\mu\right) t} d t+e^{\Lambda_{2} \xi} \int_{\xi}^{0} e^{-\left(\Lambda_{2}+\mu\right) t} d t\right. \\
& \left.=\frac{M_{3}}{\Lambda_{2}-\Lambda_{1}} \int_{0}^{+\infty} e^{\left(\mu-\Lambda_{2}\right) t} d t\right]\left|\Phi_{1}(\cdot)-\Phi_{2}(\cdot)\right|_{\mu} \\
& \quad \times\left[\frac{1}{-\Lambda_{1}-\mu}+\frac{1-e^{\left(\Lambda_{2}+\mu\right) \xi}}{\Lambda_{2}+\mu}+\frac{e^{\left(\Lambda_{2}+\mu\right) \xi}}{\Lambda_{2}-\mu}\right] \\
& \quad \times\left|\Phi_{1}(\cdot)-\Phi_{2}(\cdot)\right|_{\mu}
\end{aligned}
$$




$$
\begin{aligned}
\leq & \frac{M_{3}}{\Lambda_{2}-\Lambda_{1}} \\
& \times\left(\frac{1}{-\Lambda_{1}-\mu}+\frac{1}{\Lambda_{2}+\mu}+\frac{1}{\Lambda_{2}-\mu}\right)\left|\Phi_{1}(\cdot)-\Phi_{2}(\cdot)\right|_{\mu} \cdot
\end{aligned}
$$

If $\xi \geq 0$, we have

$$
\begin{aligned}
&\left|F_{2}\left(U_{1}(\cdot), V_{1}(\cdot)\right)(\xi)-F_{2}\left(U_{2}(\cdot), V_{2}(\cdot)\right)(\xi)\right| e^{-\mu|\xi|} \\
& \leq \frac{M_{3} e^{-\mu \xi}}{\Lambda_{2}-\Lambda_{1}} \\
& \quad \times\left[e^{\Lambda_{1} \xi} \int_{-\infty}^{0} e^{-\left(\Lambda_{1}+\mu\right) t} d t+e^{\Lambda_{1} \xi} \int_{0}^{\xi} e^{\left(\mu-\Lambda_{1}\right) t} d t\right. \\
&\left.\quad+e^{\Lambda_{2} \xi} \int_{\xi}^{+\infty} e^{\left(\mu-\Lambda_{2}\right) t} d t\right]\left|\Phi_{1}(\cdot)-\Phi_{2}(\cdot)\right|_{\mu} \\
&=\frac{M_{3}}{\Lambda_{2}-\Lambda_{1}}\left[\frac{e^{\left(\Lambda_{1}-\mu\right) \xi}}{-\Lambda_{1}-\mu}+\frac{1-e^{\left(\Lambda_{1}-\mu\right) \xi}}{\mu-\Lambda_{1}}+\frac{1}{\Lambda_{2}-\mu}\right] \\
& \quad \times\left|\Phi_{1}(\cdot)-\Phi_{2}(\cdot)\right|_{\mu} \\
& \leq \frac{M_{3}}{\Lambda_{2}-\Lambda_{1}}\left(\frac{1}{-\Lambda_{1}-\mu}+\frac{1}{\mu-\Lambda_{1}}+\frac{1}{\Lambda_{2}-\mu}\right) \\
& \times\left|\Phi_{1}(\cdot)-\Phi_{2}(\cdot)\right|_{\mu \cdot}
\end{aligned}
$$

Consequently, we conclude that

$$
\left|F_{2}\left(\Phi_{1}(\cdot)\right)-F_{2}\left(\Phi_{2}(\cdot)\right)\right|_{\mu} \leq M_{4}\left|\Phi_{1}(\cdot)-\Phi_{2}(\cdot)\right|_{\mu}
$$

where

$$
\begin{aligned}
M_{4}= & \frac{M_{3}}{\Lambda_{2}-\Lambda_{1}} \\
& \times \max \left\{\frac{1}{-\Lambda_{1}-\mu}+\frac{1}{\Lambda_{2}+\mu}+\frac{1}{\Lambda_{2}-\mu},\right. \\
& \left.\frac{1}{-\Lambda_{1}-\mu}+\frac{1}{\mu-\Lambda_{1}}+\frac{1}{\Lambda_{2}-\mu}\right\} .
\end{aligned}
$$

Thus, $F_{2}: \Gamma \rightarrow C(\mathbb{R}, \mathbb{R})$ is continuous with respect to the norm $|\cdot|_{\mu}$ in $B_{\mu}(\mathbb{R}, \mathbb{R})$. The proof is completed.

Lemma 10. $M a p F=\left(F_{1}, F_{2}\right): \Gamma \rightarrow \Gamma$ is compact with respect to the norm $|\cdot|_{\mu}$ in $B_{\mu}\left(\mathbb{R}, \mathbb{R}^{2}\right)$.

Proof. For any $\Phi(\xi)=(U(\xi), V(\xi)) \in \Gamma$, it is clear that

$$
\begin{aligned}
\frac{d}{d \xi} F_{1}(\Phi(\cdot))(\xi)= & \frac{1}{c} H_{1}(\Phi(\cdot))(\xi) \\
& -\frac{\beta_{1}}{c^{2}} \int_{-\infty}^{\xi} e^{-\left(\beta_{1} / c\right)(\xi-t)} H_{1}(\Phi(\cdot))(t) d t
\end{aligned}
$$

Since $\left|H_{1}(U, V)(\xi)\right|=\left|\left[\beta_{1}-g(V(\xi))\right] U(\xi)\right| \leq \beta_{1} U^{0}$, we have

$$
\begin{aligned}
\mid \frac{d}{d \xi} F_{1} & (\Phi(\cdot))(\xi) \mid e^{-\mu|\xi|} \\
& \leq\left|\frac{d}{d \xi} F_{1}(\Phi(\cdot))(\xi)\right| \\
& \leq \frac{\beta_{1} U^{0}}{c}+\frac{\beta_{1}}{c^{2}} e^{-\left(\beta_{1} / c\right) \xi} \int_{-\infty}^{\xi} e^{\left(\beta_{1} / c\right) t} \beta_{1} U^{0} d t \\
& =\frac{2 \beta_{1} U^{0}}{c},
\end{aligned}
$$

which implies that $\left|(d / d \xi) F_{1}(\Phi(\cdot))(\cdot)\right|_{\mu}<2 \beta_{1} U^{0} / c$. Since $\Phi(\cdot)=(U(\cdot), V(\cdot)) \in \Gamma$, we get

$$
\begin{aligned}
\left|H_{2}(U, V)(\xi)\right| & =\kappa \int_{0}^{+\infty} e^{-\kappa \eta} g(V(\xi-c \eta)) U(\xi-c \eta) d \eta \\
& \leq \kappa \int_{0}^{+\infty} e^{-\kappa \eta} \beta_{1} U^{0} d \eta+V^{0} \\
& =\beta_{1} U^{0}+V^{0} .
\end{aligned}
$$

Then,

$$
\begin{aligned}
& \left|\frac{d}{d \xi} F_{2}(\Phi(\cdot))(\xi)\right| \\
& =\frac{1}{\Lambda_{2}-\Lambda_{1}} \mid \Lambda_{1} \int_{-\infty}^{\xi} e^{\Lambda_{1}(\xi-t)} H_{2}(U, V)(t) d t \\
& \quad+\Lambda_{2} \int_{\xi}^{+\infty} e^{\Lambda_{2}(\xi-t)} H_{2}(U, V)(t) d t \mid \\
& \leq \frac{\beta_{1} U^{0}+V^{0}}{\Lambda_{2}-\Lambda_{1}} \\
& \quad \times\left[\left|\Lambda_{1}\right| \int_{-\infty}^{\xi} e^{\Lambda_{1}(\xi-t)} d t+\Lambda_{2} \int_{\xi}^{+\infty} e^{\Lambda_{2}(\xi-t)} d t\right] \\
& =\frac{2\left(\beta_{1} U^{0}+V^{0}\right)}{\Lambda_{2}-\Lambda_{1}},
\end{aligned}
$$

which implies that $\left|(d / d \xi) F_{2}(\Phi(\cdot))(\cdot)\right|_{\mu}<2\left(\beta_{1} U^{0}+V^{0}\right) /$ $\left(\Lambda_{2}-\Lambda_{1}\right)$. Consequently, $\left|(d / d \xi) F_{1}(\Phi(\cdot))(\cdot)\right|_{\mu}$ and $\left|(d / d \xi) F_{2}(\Phi(\cdot))(\cdot)\right|_{\mu}$ are bounded, which shows that $F(\Gamma)$ is uniformly bounded and equicontinuous with respect to the $\operatorname{norm}|\cdot|_{\mu}$ in $B_{\mu}\left(\mathbb{R}, \mathbb{R}^{2}\right)$.

Furthermore, for any positive integer $n$, define

$$
\begin{aligned}
& F^{n}(U(\cdot), V(\cdot))(\xi) \\
& \quad= \begin{cases}F(U(\cdot), V(\cdot))(\xi), & \xi \in[-n, n], \\
F(U(\cdot), V(\cdot))(-n), & \xi \in(-\infty,-n], \\
F(U(\cdot), V(\cdot))(n), & \xi \in[n,+\infty] .\end{cases}
\end{aligned}
$$


Obviously, for fixed $n, F^{n}(\Gamma)$ is uniformly bounded and equicontinuous with respect to the norm $|\cdot|_{\mu}$ in $B_{\mu}\left(\mathbb{R}, \mathbb{R}^{2}\right)$, which implies that $F^{n}: \Gamma \rightarrow \Gamma$ is a compact operator. Since

$$
\begin{aligned}
\left|F_{1}(U(\cdot), V(\cdot))(\xi)\right| \leq & \frac{1}{c} \int_{-\infty}^{\xi} e^{-\left(\beta_{1} / c\right)(\xi-t)} \beta_{1} U^{0} d t=U^{0}, \\
\left|F_{2}(U(\cdot), V(\cdot))(\xi)\right| \leq & \frac{\beta_{1} U^{0}+V^{0}}{\Lambda_{2}-\Lambda_{1}} \\
& \times\left\{\int_{-\infty}^{\xi} e^{\Lambda_{1}(\xi-t)} d t+\int_{\xi}^{+\infty} e^{\Lambda_{2}(\xi-t)} d t\right\} \\
= & \frac{\beta_{1} U^{0}+V^{0}}{\left|\Lambda_{1}\right| \Lambda_{2}},
\end{aligned}
$$

we have that

$$
\begin{aligned}
& \left|F_{1}^{n}(U(\cdot), V(\cdot))(\cdot)-F_{1}(U(\cdot), V(\cdot))(\cdot)\right|_{\mu} \\
& \quad=\sup _{\xi \in \mathbb{R}}\left|F_{1}^{n}(U(\cdot), V(\cdot))(\xi)-F_{1}(U(\cdot), V(\cdot))(\xi)\right| e^{-\mu|\xi|} \\
& \quad=\sup _{\xi \in(-\infty,-n] \cup[n,+\infty)} \mid F_{1}^{n}(U(\cdot), V(\cdot))(\xi) \\
& \quad-F_{1}(U(\cdot), V(\cdot))(\xi) \mid e^{-\mu|\xi|} \\
& \leq 2 U^{0} e^{-\mu n} \longrightarrow 0, \quad \text { as } n \longrightarrow+\infty .
\end{aligned}
$$

Similarly, we can prove that $\mid F_{2}^{n}(U(\cdot), V(\cdot))(\cdot)-F_{2}(U(\cdot)$, $V(\cdot))\left.(\cdot)\right|_{\mu} \rightarrow 0$ when $n \rightarrow+\infty$. Thus, $\mid F^{n}(U(\cdot), V(\cdot))(\cdot)-$ $\left.F(U(\cdot), V(\cdot))(\cdot)\right|_{\mu} \rightarrow 0$ when $n \rightarrow+\infty$. By Proposition 2.1 in Zeilder [40], we have that $F^{n}$ converges to $F$ in $\Gamma$ with respect to the norm $|\cdot|_{\mu}$. Consequently, $F: \Gamma \rightarrow \Gamma$ is compact with respect to the norm $|\cdot|_{\mu}$. The proof is completed.

Proof of Theorem 1. Combination of Schauder's fixed point theorem and Lemmas 8, 9, and 10 shows that there exists a nonnegative traveling wave solution $\left(U_{c}(\cdot), V_{c}(\cdot)\right) \in \Gamma$ such that $\left(U_{c}(\xi), V_{c}(\xi)\right) \rightarrow\left(U^{0}, 0\right)$ when $\xi \rightarrow-\infty$. By the definition of $\underline{U}(\xi)$ and $\underline{V}(\xi)$, there is a $\xi_{0}<0$ such that $\underline{U}(\xi)>0$ and $\underline{V}(\xi)>0$ when $\xi<\xi_{0}$. Therefore, if $\xi<\xi_{0}$, we have that $c \bar{U}_{c}^{\prime}=-g\left(V_{c}(\xi)\right) U(\xi)<0$ which implies that $U^{0}>U^{1} \geq 0$. It is clear that $U_{c}(\xi)$ is nonincreasing in $\mathbb{R}$.

From Lemma 2 and system (18), it follows that

$$
\begin{aligned}
& c U_{c}^{\prime}=-g\left(V_{c}(\xi)\right) U_{c}(\xi) \\
& W_{c}(\xi)=\kappa \int_{0}^{+\infty} e^{-\kappa \eta} g\left(V_{c}(\xi-c \eta)\right) U_{c}(\xi-c \eta) d \eta \\
& c V_{c}^{\prime}=V_{c}^{\prime \prime}+W_{c}(\xi)-V_{c}(\xi) .
\end{aligned}
$$

By the first and second equation of (69), we have

$$
\begin{aligned}
\int_{-\infty}^{+\infty} & W_{c}(\xi) d \xi \\
& =-\kappa c \int_{-\infty}^{+\infty} \int_{0}^{+\infty} e^{-\kappa \eta} U_{c}^{\prime}(\xi-c \eta) d \eta d \xi \\
& =-\kappa c \int_{0}^{+\infty} e^{-\kappa \eta} \int_{-\infty}^{+\infty} U_{c}^{\prime}(\xi-c \eta) d \xi d \eta \\
& =\left(U^{0}-U^{1}\right) \kappa c \int_{0}^{+\infty} e^{-\kappa \eta} d \eta \\
& =c\left(U^{0}-U^{1}\right) .
\end{aligned}
$$

Since the traveling wave solution $\left(U_{c}(\cdot), V_{c}(\cdot)\right) \in \Gamma$ and is the fixed point of the operator $F$, thus $\left(U_{c}(\xi), V_{c}(\xi)\right)=$ $F\left(U_{c}(\cdot), V_{c}(\cdot)\right)(\xi)$. The proof of Lemma 10 shows that $V_{c}^{\prime}(\xi)=$ $F_{2}^{\prime}\left(U_{c}(\cdot), V_{c}(\cdot)\right)(\xi)$ is bounded. Using L'Hopital principal shows that

$$
V_{c}^{\prime}(-\infty)=F_{2}^{\prime}\left(U_{c}(\cdot), V_{c}(\cdot)\right)(-\infty)=0 .
$$

Integrating the third equation of (69) from $-\infty$ to $\xi$ yields

$$
c V_{c}(\xi)=V_{c}^{\prime}(\xi)+\int_{-\infty}^{\xi} W_{c}(\eta) d \eta-\int_{-\infty}^{\xi} V_{c}(\eta) d \eta .
$$

Then, the boundedness of $V_{c}(\xi)$ and $V_{c}^{\prime}(\xi)$ implies $\int_{-\infty}^{+\infty} V_{c}(\eta) d \eta<+\infty$, which shows that $\lim _{\xi \rightarrow+\infty} V_{c}(\xi)=0$ and $\int_{-\infty}^{+\infty} W_{c}(\eta) d \eta=\int_{-\infty}^{+\infty} V_{c}(\eta) d \eta=c\left(U^{0}-U^{1}\right)$.

Next, we prove $0 \leq V_{c}(\xi) \leq U^{0}-U^{1}$, which is motivated by Wang and $\mathrm{Wu}$ [34]. Define

$$
R(\xi)=\frac{1}{c} \int_{-\infty}^{\xi} V_{c}(\eta) d \eta+\frac{1}{c} \int_{\xi}^{+\infty} e^{c(\xi-\eta)} V_{c}(\eta) d \eta
$$

It is clear that $R(\xi)$ satisfies

$$
c R^{\prime}=R^{\prime \prime}+R(\xi),
$$

$R(-\infty)=0$, and $R(+\infty)=U^{0}-U^{1}$. Denote $N(\xi)=V_{c}(\xi)+$ $R(\xi)$, which satisfies

$$
c N^{\prime}=N^{\prime \prime}+W_{c}(\xi) .
$$

Due to $N^{\prime}(+\infty)=0$, it follows that

$$
N^{\prime}(\xi)=\int_{\xi}^{+\infty} e^{c(\xi-\eta)} W_{c}(\eta) d \eta \geq 0
$$

which implies that $N(\xi)$ is nondecreasing in $\mathbb{R}$. Since $N(-\infty)=0$ and $N(+\infty)=U^{0}-U^{1}$, we have $0 \leq V_{c}(\xi) \leq$ $U^{0}-U^{1}$ for all $\xi \in \mathbb{R}$. The proof is completed.

\section{Nonexistence of Traveling Wave Solutions}

In this section, we give the conditions on which system (5) has no traveling wave solutions. 
Theorem 11. (I) Assume $U^{0}>1 / g^{\prime}(0)$. Then, for any $0<$ $c<c^{*}$, system (5) has no nonnegative nontrivial traveling wave solutions $(U(y+c \tau), W(y+c \tau), V(y+c \tau))$ satisfying boundary condition (8).

(II) Suppose $0<U^{0} \leq 1 / g^{\prime}(0)$. Then, for any $c>0$, system (5) has no traveling wave solutions $(U(y+c \tau), W(y+c \tau), V(y+$ $c \tau)$ ) satisfying boundary condition (8).

To prove (I) by two-sided Laplace transform, the following lemma about the exponential decrease of traveling wave solutions is needed.

Lemma 12. Assume $U^{0}>1 / g^{\prime}(0)$. If $(U(y+c \tau), W(y+$ $c \tau), V(y+c \tau))$ is a nonnegative nontrivial traveling wave solution of (5) satisfying boundary condition (8), there exists a positive constant $\alpha$ such that

$$
\sup _{\xi \in \mathbb{R}}\left\{W(\xi) e^{-\alpha \xi}\right\}<+\infty, \quad \sup _{\xi \in \mathbb{R}}\left\{V(\xi) e^{-\alpha \xi}\right\}<+\infty .
$$

Proof. Substituting traveling wave profile

$$
u_{2}(y, \tau)=W(\xi), \quad u_{3}(y, \tau)=V(\xi), \quad \xi=y+c \tau
$$

into the second and third equations of (5), it follows that

$$
\begin{gathered}
c W^{\prime}=\kappa[g(V) U-W], \\
c V^{\prime}=V^{\prime \prime}+W-V .
\end{gathered}
$$

Setting $V^{\prime}=Z$, we have

$$
\begin{gathered}
c W^{\prime}=\kappa[g(V) U-W], \\
V^{\prime}=Z, \\
Z^{\prime}=c Z+V-W .
\end{gathered}
$$

Furthermore, system (80) can be written as

$$
\psi^{\prime}=A \psi+f(\xi, \psi),
$$

where

$$
\begin{aligned}
& A=\left[\begin{array}{ccc}
-\frac{\kappa}{c} & \frac{\kappa g^{\prime}(0) U^{0}}{c} & 0 \\
0 & 0 & 1 \\
-1 & 1 & c
\end{array}\right], \\
& \psi=\left[\begin{array}{l}
W \\
V \\
Z
\end{array}\right], \\
& f(\xi, \psi)=\left[\frac{\kappa U^{0} \bar{G}(V)}{c}+\frac{\kappa\left(U-U^{0}\right) g(V)}{c}\right] \text {, }
\end{aligned}
$$

and $\bar{G}(V)=g(V)-g^{\prime}(0) V$. Obviously, $\bar{G}^{\prime}(0)=0$. Since

$$
\lim _{\xi \rightarrow-\infty} U(\xi)=U^{0}, \quad \lim _{\xi \rightarrow-\infty} \psi(\xi)=0,
$$

and $g^{\prime}(V)$ is bounded in $[0,+\infty)$, for any small constant $\varepsilon>0$, there exists $\xi_{0} \in \mathbb{R}$ such that

$$
\left\|f\left(\xi, \psi_{1}\right)-f\left(\xi, \psi_{2}\right)\right\| \leq \varepsilon\left\|\psi_{1}-\psi_{2}\right\|
$$

for all $\xi \leq \xi_{0}$.

Since $(U(\xi), W(\xi), V(\xi))$ is the solution of (79) if and only if $(U(\xi-\eta), W(\xi-\eta), V(\xi-\eta))$ is the solution of (79) for any $\eta \in \mathbb{R}$ and they satisfy the same boundary condition at $\pm \infty$, we can select large $\eta$ such that (84) holds with $\xi_{0}=0$ and $(U(\xi), W(\xi), V(\xi))$ being replaced by $(U(\xi-\eta), W(\xi-\eta), V(\xi-$ $\eta))$. Therefore, we suppose $\xi_{0}=0$.

Calculations show that the eigenfunction of $A$ is $H(\lambda)$ defined by (21). Then, by Lemma 4 , there exists constant matrix $C$ such that

$$
C^{-1} A C=B=\left[\begin{array}{cc}
\lambda_{0} & 0 \\
0 & Q
\end{array}\right],
$$

where $\lambda_{0}<0$ and the eigenvalues $\lambda_{1}, \lambda_{2}$ of $2 \times 2$ matrix $Q$ have positive real parts. Setting $\psi=C \varphi$, the system (81) then has the form

$$
\frac{d \varphi}{d \xi}=B \varphi+G(\xi, \varphi)
$$

Obviously, $\lim _{\xi \rightarrow-\infty} \varphi(\xi)=0$ and $G(\xi, \varphi)=C^{-1} f(\xi, C \psi)$ satisfies (84). Denote

$$
\Phi_{1}(\xi)=\left[\begin{array}{cc}
e^{\lambda_{0} \xi} & 0 \\
0 & 0
\end{array}\right], \quad \Phi_{2}(\xi)=\left[\begin{array}{cc}
0 & 0 \\
0 & e^{\mathrm{Q} \xi}
\end{array}\right] .
$$

Then, $\Phi_{1}^{\prime}=B \Phi_{1}, \Phi_{2}^{\prime}=B \Phi_{2}$, and

$$
e^{B \xi}=\Phi_{1}(\xi)+\Phi_{2}(\xi) .
$$

It is not difficult to see that we can choose positive constants $\alpha$ and $L$ such that

$$
\begin{array}{cc}
\left\|\Phi_{1}(\xi)\right\| \leq L e^{-2 \alpha \xi} & \text { for any } \xi \geq 0, \\
\left\|\Phi_{2}(\xi)\right\| \leq L e^{2 \alpha \xi} & \text { for any } \xi \leq 0 .
\end{array}
$$

It is clear that (86) is equivalent to

$$
\begin{aligned}
& u_{1}(\xi)=e^{\lambda_{0} \xi} u_{1}(0)+\int_{0}^{\xi} e^{\lambda_{0}(\xi-\eta)} G_{1}(\eta, u(\eta)) d \eta, \\
& u_{2}(\xi)=e^{Q \xi} u_{2}(0)+\int_{0}^{\xi} e^{Q(\xi-\eta)} G_{2}(\eta, u(\eta)) d \eta,
\end{aligned}
$$

where

$$
\begin{gathered}
u=\left(u_{1}, u_{2}^{T}\right)^{T}=\varphi, \quad u_{1}=\varphi_{1}, \quad u_{2}=\left(\varphi_{2}, \varphi_{3}\right)^{T}, \\
\left(G_{1}(\eta, u), G_{2}(\eta, u)^{T}\right)^{T}=G(\eta, u) .
\end{gathered}
$$

Since $\lim _{\xi \rightarrow-\infty} u(\xi)=0$, then multiplying the first equality of (90) by $e^{-\lambda_{0} \xi}$ and setting $\xi \rightarrow-\infty$ yield

$$
u_{1}(0)+\int_{0}^{-\infty} e^{-\lambda_{0} \eta} G_{1}(\eta, u(\eta)) d \eta=0
$$


or

$$
u_{1}(0)=\int_{-\infty}^{0} e^{-\lambda_{0} \eta} G_{1}(\eta, u(\eta)) d \eta .
$$

Thus, (90) has the form

$$
\begin{aligned}
u(\xi)= & \int_{-\infty}^{\xi} \Phi_{1}(\xi-\eta) G(\eta, u(\eta)) d \eta \\
& +\Phi_{2}(\xi) u_{0}-\int_{\xi}^{0} \Phi_{2}(\xi-\eta) G(\eta, u(\eta)) d \eta
\end{aligned}
$$

where $u_{0}=\left(0, u_{2}(0)^{T}\right)^{T}$.

To study the properties of $u(\xi)$, we need to construct a functional sequence. Define

$$
\begin{gathered}
u^{0}(\xi)=0 \\
u^{j+1}(\xi)=\int_{-\infty}^{\xi} \Phi_{1}(\xi-\eta) G\left(\eta, u^{j}(\eta)\right) d \eta+\Phi_{2}(\xi) u_{0} \\
-\int_{\xi}^{0} \Phi_{2}(\xi-\eta) G\left(\eta, u^{j}(\eta)\right) d \eta
\end{gathered}
$$

for $\xi \leq 0$. Obviously, we have

$$
\left\|u^{1}(\xi)-u^{0}(\xi)\right\| \leq\left\|\Phi_{2}(\xi) u_{0}\right\| \leq L\left\|u_{0}\right\| e^{\alpha \xi}
$$

for any $\xi \leq 0$. Assume that the induction hypothesis

$$
\left\|u^{j}(\xi)-u^{j-1}(\xi)\right\| \leq \frac{L\left\|u_{0}\right\| e^{\alpha \xi}}{2^{j-1}}
$$

holds for $j=1,2, \ldots, m$ and $\xi \leq 0$. Then, using the condition (84) satisfied by the function $G$, it follows from the induction hypothesis that for $\xi \leq 0$ we have

$$
\begin{aligned}
\left\|u^{m+1}(\xi)-u^{m}(\xi)\right\| & \\
\leq & \int_{-\infty}^{\xi}\left\|\Phi_{1}(\xi-\eta)\right\| \varepsilon\left\|u^{m}(\eta)-u^{m-1}(\eta)\right\| d \eta \\
& +\int_{\xi}^{0}\left\|\Phi_{2}(\xi-\eta)\right\| \varepsilon\left\|u^{m}(\eta)-u^{m-1}(\eta)\right\| d \eta \\
\leq & \varepsilon \int_{-\infty}^{\xi} L e^{-2 \alpha(\xi-\eta)} \frac{L\left\|u_{0}\right\| e^{\alpha \eta}}{2^{m-1}} d \eta \\
& +\varepsilon \int_{\xi}^{0} L e^{2 \alpha(\xi-\eta)} \frac{L\left\|u_{0}\right\| e^{\alpha \eta}}{2^{m-1}} d \eta \\
\leq & \frac{\varepsilon L^{2}\left\|u_{0}\right\| e^{\alpha \xi}}{3 \alpha 2^{m-1}}+\frac{\varepsilon L^{2}\left\|u_{0}\right\| e^{\alpha \xi}}{\alpha 2^{m-1}} \\
= & \frac{8 \varepsilon L}{3 \alpha} \frac{L\left\|u_{0}\right\| e^{\alpha \xi}}{2^{m}} .
\end{aligned}
$$

Setting $\varepsilon \leq 3 \alpha /(8 L)$ yields

$$
\left\|u^{m+1}(\xi)-u^{m}(\xi)\right\| \leq \frac{L\left\|u_{0}\right\| e^{\alpha \xi}}{2^{m}} .
$$

It then follows by induction that (97) holds for all $j=$ $1,2,3, \ldots$ and $\xi<0$. Thus, for any $n>m>N$ and $\xi<0$, we have

$$
\begin{aligned}
\left\|u^{n}(\xi)-u^{m}(\xi)\right\| & \leq \sum_{j=N}^{\infty}\left\|u^{j+1}(\xi)-u^{j}(\xi)\right\| \\
& \leq L\left\|u_{0}\right\| \sum_{j=N}^{\infty} \frac{1}{2^{j}}=\frac{L\left\|u_{0}\right\|}{2^{N-1}}
\end{aligned}
$$

which implies that $\left\{u^{j}(\xi)\right\}$ is a Cauchy sequence of continuous functions. It follows that

$$
\lim _{j \rightarrow \infty} u^{j}(\xi)=\bar{u}(\xi)
$$

uniformly for all $\xi<0$. Taking the limit of both sides of (95), it follows from the uniform convergence that the continuous function $\bar{u}(\xi)$ satisfies the integral equation (94). Then, the uniqueness of solution of (94) implies $u(\xi)=\bar{u}(\xi)$. Consequently, (97) and

$$
u^{m}(\xi)=\sum_{j=1}^{m}\left[u^{j}(\xi)-u^{j-1}(\xi)\right]
$$

show that

$$
\|u(\xi)\| \leq 2 L\left\|u_{0}\right\| e^{\alpha \xi}
$$

for any $\xi<0$. Since $\lim _{\xi \rightarrow+\infty} u(\xi)=0$, it follows $\left\|u(\xi) e^{-\alpha \xi}\right\|<$ $+\infty$. Thus,

$$
\sup _{\xi \in \mathbb{R}}\left\{I_{u}(\xi) e^{-\alpha \xi}\right\}<+\infty, \quad \sup _{\xi \in \mathbb{R}}\left\{I_{t}(\xi) e^{-\alpha \xi}\right\}<+\infty
$$

and the proof is completed.

Proof of Theorem 11(I). Assume (5) has a traveling wave solution $(U(y+c \tau), W(y+c \tau), V(y+c \tau))$ satisfying boundary condition (8). From the first equation of (18), it follows

$$
\begin{gathered}
U(\xi)=U^{0} e^{-(1 / c) \int_{-\infty}^{\xi} g(V(\eta)) d \eta}, \\
U^{0}-U(\xi)=U^{0}\left[1-e^{-(1 / c) \int_{-\infty}^{\xi} g(V(\eta)) d \eta}\right] .
\end{gathered}
$$

By Lemma 12, there exists a positive constant $\alpha^{\prime}$ such that

$$
\sup _{\xi \in \mathbb{R}}\left\{\left[U^{0}-U(\xi)\right] e^{-\alpha^{\prime} \xi}\right\}<+\infty
$$

For $0<\lambda<\alpha$, define two-sided Laplace transform as follows:

$$
\mathscr{L}[V(\cdot)](\lambda):=\int_{-\infty}^{+\infty} e^{-\lambda \xi} V(\xi) d \xi
$$

Obviously, there exists $\lambda^{*}>0$ and $\mathscr{L}[V(\cdot)](\lambda)$ is increasing in $\left[0, \lambda^{*}\right)$ such that $\lambda^{*}<+\infty$ satisfying $\lim _{\lambda \rightarrow \lambda^{*-}} \mathscr{L}[V(\cdot)](\lambda)=$ $+\infty$ or $\lambda^{*}=+\infty$. 
Furthermore, we have

$$
\begin{aligned}
& \mathscr{L}\left[\int_{0}^{+\infty} e^{-\kappa \eta} J(V(\cdot-c \eta)) d \eta\right](\lambda) \\
& \quad=\int_{0}^{+\infty} e^{-\kappa \eta} \int_{-\infty}^{+\infty} e^{-\lambda \xi} J(V(\cdot-c \eta)) d \xi d \eta \\
& \quad=\int_{0}^{+\infty} e^{-\kappa \eta} \int_{-\infty}^{+\infty} e^{-\lambda(t+c \eta)} J(V(t)) d t d \eta \\
& \quad=\int_{0}^{+\infty} e^{-(\kappa+c \lambda) \eta} \int_{-\infty}^{+\infty} e^{-\lambda t} J(V(t)) d t d \eta \\
& =\frac{1}{\kappa+c \lambda} \mathscr{L}[J(V(\cdot))](\lambda) .
\end{aligned}
$$

The second equality of (18) can be rewritten as

$$
L[V(\cdot)](\xi)=\int_{0}^{+\infty} \kappa e^{-\kappa \eta} Q(\xi-c \eta) d \eta
$$

where

$$
\begin{aligned}
& L[V(\cdot)](\xi):= V^{\prime \prime}(\xi)-c V^{\prime}(\xi)+\kappa g^{\prime}(0) U^{0} \\
& \times \int_{0}^{+\infty} e^{-\kappa \eta} V(\xi-c \eta) d \eta-V(\xi), \\
& Q(\xi):=\left[g^{\prime}(0) V(\xi) U^{0}-g(V(\xi)) U(\xi)\right] .
\end{aligned}
$$

Denote $\mathscr{G}(V):=g^{\prime}(0) V-g(V)$, from which it follows that $\mathscr{G}^{\prime}(0)=0$. Since $g(V)$ satisfies hypothesis (A1), we have $\mathscr{G}(V) \geq 0$ for any $V \geq 0$.

Taking two-sided Laplace transform of (109), we have

$$
\Theta(\lambda) \mathscr{L}[V(\cdot)](\lambda)=\frac{\kappa}{\kappa+c \lambda} \mathscr{L}[Q(\cdot)](\lambda)
$$

If $\lambda^{*}<+\infty$, it is clear that $\mathscr{L}[V(\cdot)]\left(\lambda^{*}-\right)=+\infty$. Since

$$
Q(\xi)=g^{\prime}(0) V(\xi)\left[U^{0}-U(\xi)\right]+\mathscr{G}(V(\xi)) U(\xi)
$$

Lemma 12, inequality (106), and $G^{\prime}(0)=0$ imply $\mathscr{L}[Q(\cdot)]\left(\lambda^{*}\right)<+\infty$. However, Lemma 4 shows that $0<$ $\Theta\left(\lambda^{*}\right)<+\infty$, which contradicts (111). Hence, we have $\lambda^{*}=$ $+\infty$. Furthermore, (111) can be rewritten as

$$
\int_{-\infty}^{+\infty} e^{-\lambda \xi}\left[\Theta(\lambda) V(\xi)-\int_{0}^{+\infty} \kappa e^{-\kappa \eta} Q(\xi-c \eta) d \eta\right] d \xi=0
$$

Since $\lim _{\lambda \rightarrow+\infty} \Theta(\lambda)=+\infty$, it is impossible that the above equality holds, again a contradiction.

Proof of Theorem 11(II). Suppose $(U(y+c \tau), W(y+c \tau), V(y+$ $c \tau)$ ) is a traveling wave solution of system (5) satisfying boundary condition (8). Then, by the proof of Theorem 1, it is clear that $\int_{-\infty}^{+\infty} V(\xi) d \xi=\int_{-\infty}^{+\infty} H_{2}(U, V)(\xi) d \xi$. Furthermore,

$$
\begin{aligned}
\int_{-\infty}^{+\infty} & H_{2}(U, V)(\xi) d \xi \\
& =\int_{-\infty}^{+\infty} \int_{0}^{+\infty} \kappa e^{-\kappa \eta} g(V(\xi-c \eta)) U(\xi-c \eta) d \eta d \xi \\
& <\int_{-\infty}^{+\infty} \int_{0}^{+\infty} \kappa e^{-\kappa \eta} g^{\prime}(0) V(\xi-c \eta) U^{0} d \eta d \xi \\
& =g^{\prime}(0) U^{0} \int_{0}^{+\infty} \kappa e^{-\kappa \eta} \int_{-\infty}^{+\infty} V(\xi-c \eta) d \xi d \eta \\
& \leq \int_{0}^{+\infty} \kappa e^{-\kappa \eta} \int_{-\infty}^{+\infty} V(\xi) d \xi d \eta \\
& =\int_{-\infty}^{+\infty} V(\xi) d \xi
\end{aligned}
$$

which is a contradiction. The proof is completed.

\section{Conflict of Interests}

The authors declare that there is no conflict of interests regarding the publication of this paper.

\section{Acknowledgments}

The authors are supported by the Fundamental Research Funds for the Central Universities (XDJK2012C042) and NSFC Grants (11201380).

\section{References}

[1] Centers for Disease Control and Prevention, USA, http://www .cdc.gov/cholera/general/.

[2] World Health Organization, http://www.who.int/csr/don/archive/disease/cholera/en/.

[3] V. Capasso and S. L. Paveri-Fontana, "Mathematical model for the 1973 cholera epidemic in the European Mediterranean region," Revue d'Epidemiologie et de Sante Publique, vol. 27, no. 2, pp. 121-132, 1979.

[4] C. T. Codeço, "Endemic and epidemic dynamics of cholera: the role of the aquatic reservoir," BMC Infectious Diseases, vol. 1, article 1, 2001.

[5] K. T. Goh, S. H. Teo, S. Lam, and M. K. Ling, "Person-to-person transmission of cholera in a psychiatric hospital," Journal of Infection, vol. 20, no. 3, pp. 193-200, 1990.

[6] J. H. Tien and D. J. D. Earn, "Multiple transmission pathways and disease dynamics in a waterborne pathogen model," Bulletin of Mathematical Biology, vol. 72, no. 6, pp. 1506-1533, 2010.

[7] A. A. Weil, A. I. Khan, F. Chowdhury et al., "Clinical outcomes in household contacts of patients with cholera in Bangladesh," Clinical Infectious Diseases, vol. 49, no. 10, pp. 1473-1479, 2009.

[8] J. R. Andrews and S. Basu, "Transmission dynamics and control of cholera in Haiti: an epidemic model," The Lancet, vol. 377, no. 9773, pp. 1248-1255, 2011. 
[9] D. M. Hartley, J. G. Morris Jr., and D. L. Smith, "Hyperinfectivity: a critical element in the ability of $V$. cholerae to cause epidemics?" PLoS Medicine, vol. 3, no. 1, pp. 63-69, 2006.

[10] M. A. Jensen, S. M. Faruque, J. J. Mekalanos, and B. R. Levin, "Modeling the role of bacteriophage in the control of cholera outbreaks," Proceedings of the National Academy of Sciences of the United States of America, vol. 103, no. 12, pp. 4652-4657, 2006.

[11] Z. Mukandavire, S. Liao, J. Wang, H. Gaff, D. L. Smith, and J. G. Morris Jr., "Estimating the reproductive numbers for the 2008-2009 cholera outbreaks in Zimbabwe," Proceedings of the National Academy of Sciences of the United States of America, vol. 108, no. 21, pp. 8767-8772, 2011.

[12] R. P. Sanches, C. P. Ferreira, and R. A. Kraenkel, "The role of immunity and seasonality in cholera epidemics," Bulletin of Mathematical Biology, vol. 73, no. 12, pp. 2916-2931, 2011.

[13] Z. Shuai and P. van den Driessche, "Global dynamics of cholera models with differential infectivity," Mathematical Biosciences, vol. 234, no. 2, pp. 118-126, 2011.

[14] Z. Shuai, J. H. Tien, and P. V. V. Driessche, "Cholera Models with hyperinfectivity and temporary immunity," Bulletin of Mathematical Biology, vol. 74, no. 10, pp. 2423-2445, 2012.

[15] J. P. Tian and J. Wang, "Global stability for cholera epidemic models," Mathematical Biosciences, vol. 232, no. 1, pp. 31-41, 2011.

[16] A. Mutreja, D. W. Kim, N. R. Thomson et al., "Evidence for several waves of global transmission in the seventh cholera pandemic," Nature, vol. 477, no. 7365, pp. 462-465, 2011.

[17] M. Emch, C. Feldacker, M. S. Islamand, and M. Ali, "Global climate and infectious disease: the cholera paradigm," Science, vol. 274, no. 5295, pp. 2025-2031, 1996.

[18] S. M. Faruque, M. J. Islam, Q. S. Ahmad et al., "Self-limiting nature of seasonal cholera epidemics: role of host-mediated amplification of phage," Proceedings of the National Academy of Sciences of the United States of America, vol. 102, no. 17, pp. 61196124, 2005.

[19] V. Capasso and K. Kunisch, "A reaction-diffiusion system arising in modelling man- environment diseases," Quarterly of Applied Mathematics, vol. 46, no. 3, pp. 431-450, 1988.

[20] V. Capasso and L. Maddalena, "Convergence to equilibrium states for a reaction-diffusion system modelling the spatial spread of a class of bacterial and viral diseases," Journal of Mathematical Biology, vol. 13, no. 2, pp. 173-184, 1981.

[21] V. Capasso and R. E. Wilson, "Analysis of a reaction-diffusion system modeling man-environment-man epidemics," SIAM Journal on Applied Mathematics, vol. 57, no. 2, pp. 327-346, 1997.

[22] S. Aniţa and V. Capasso, "Stabilization of a reaction-diffusion system modelling a class of spatially structured epidemic systems via feedback control," Nonlinear Analysis: Real World Applications, vol. 13, no. 2, pp. 725-735, 2012.

[23] E. Bertuzzo, S. Azaele, A. Maritan, M. Gatto, I. RodriguezIturbe, and A. Rinaldo, "On the space-time evolution of a cholera epidemic," Water Resources Research, vol. 44, no. 1, Article ID W01424, 2008.

[24] E. Bertuzzo, R. Casagrandi, M. Gatto, I. Rodriguez-Iturbe, and A. Rinaldo, "On spatially explicit models of cholera epidemics," Journal of the Royal Society Interface, vol. 7, no. 43, pp. 321-333, 2010.

[25] L. Mari, E. Bertuzzo, L. Righetto et al., "Modelling cholera epidemics: the role of waterways, human mobility and sanitation," Journal of the Royal Society Interface, vol. 9, no. 67, pp. 376-388, 2012.
[26] O. Diekmann, "Thresholds and travelling waves for the geographical spread of infection," Journal of Mathematical Biology, vol. 6, no. 2, pp. 109-130, 1978.

[27] M. Lewis, J. Rencławowicz, and P. van den Driessche, "Traveling waves and spread rates for a west nile virus model," Bulletin of Mathematical Biology, vol. 68, no. 1, pp. 3-23, 2006.

[28] J. Radcliffe and L. Rass, "The asymptotic speed of propagation of the deterministic non-reducible n-type epidemic," Journal of Mathematical Biology, vol. 23, no. 3, pp. 341-359, 1986.

[29] X.-Q. Zhao and W. Wang, "Fisher waves in an epidemic model," Discrete and Continuous Dynamical Systems B, vol. 4, no. 4, pp. 1117-1128, 2004.

[30] D. Xu and X.-Q. Zhao, "Bistable waves in an epidemic model," Journal of Dynamics and Differential Equations, vol. 16, no. 3, pp. 679-707, 2004.

[31] Y. Jin and X.-Q. Zhao, "Bistable waves for a class of cooperative reaction-diffiusion systems," Journal of Biological Dynamics, vol. 2, no. 2, pp. 196-207, 2008.

[32] C.-H. Hsu and T.-S. Yang, "Existence, uniqueness, monotonicity and asymptotic behaviour of travelling waves for epidemic models," Nonlinearity, vol. 26, no. 1, pp. 121-139, 2013.

[33] W.-T. Li, G. Lin, and S. Ruan, "Existence of travelling wave solutions in delayed reaction-diffusion systems with applications to diffusion-competition systems," Nonlinearity, vol. 19, no. 6, pp. 1253-1273, 2006.

[34] Z.-C. Wang and J. Wu, "Travelling waves of a diffiusive KermackCMcKendrick epidemic model with non-local delayed transmission," Proceedings of the Royal Society A: Mathematical, Physical and Engineering Sciences, vol. 466, no. 2113, pp. 237-261, 2010.

[35] Z.-C. Wang, W.-T. Li, and S. Ruan, "Traveling fronts in monostable equations with nonlocal delayed effects," Journal of Dynamics and Differential Equations, vol. 20, no. 3, pp. 573-607, 2008.

[36] Z.-C. Wang, W.-T. Li, and S. Ruan, "Entire solutions in bistable reaction-diffusion equations with nonlocal delayed nonlinearity," Transactions of the American Mathematical Society, vol. 361, no. 4, pp. 2047-2084, 2009.

[37] J. Carr and A. Chmaj, "Uniqueness of travelling waves for nonlocal monostable equations," Proceedings of the American Mathematical Society, vol. 132, no. 8, pp. 2433-2439, 2004.

[38] L. Perko, Diffierential Equations and Dynamical Systems, Springer, New York, NY, US, 2001.

[39] R. S. Irving, Integers, Polynomials, and Rings, Springer, New York, NY, USA, 2004.

[40] E. Zeilder, Nonlinear Functional Analysis and Its Applications I, Springer, New York, NY, USA, 1986. 


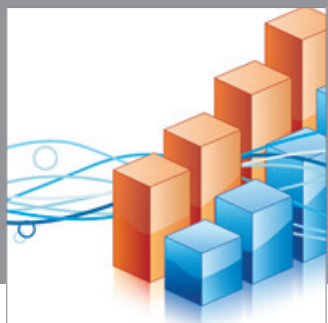

Advances in

Operations Research

mansans

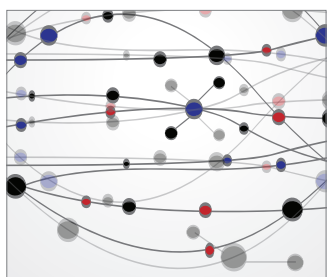

The Scientific World Journal
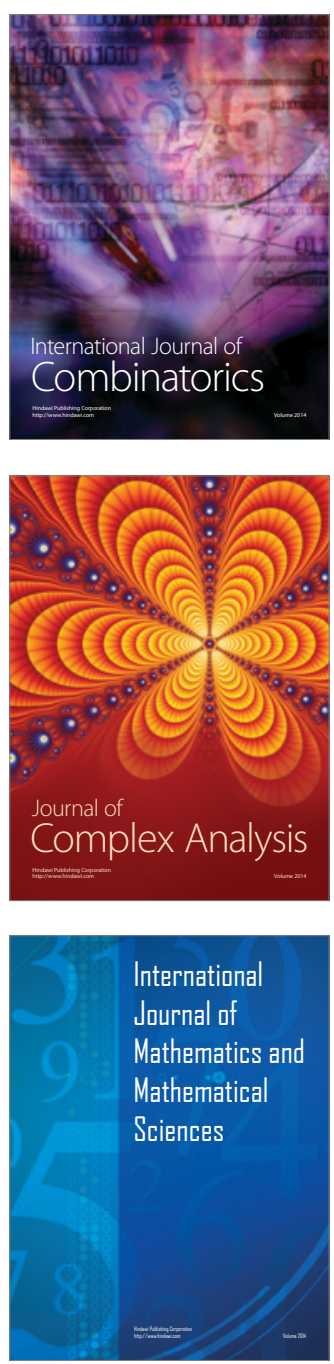
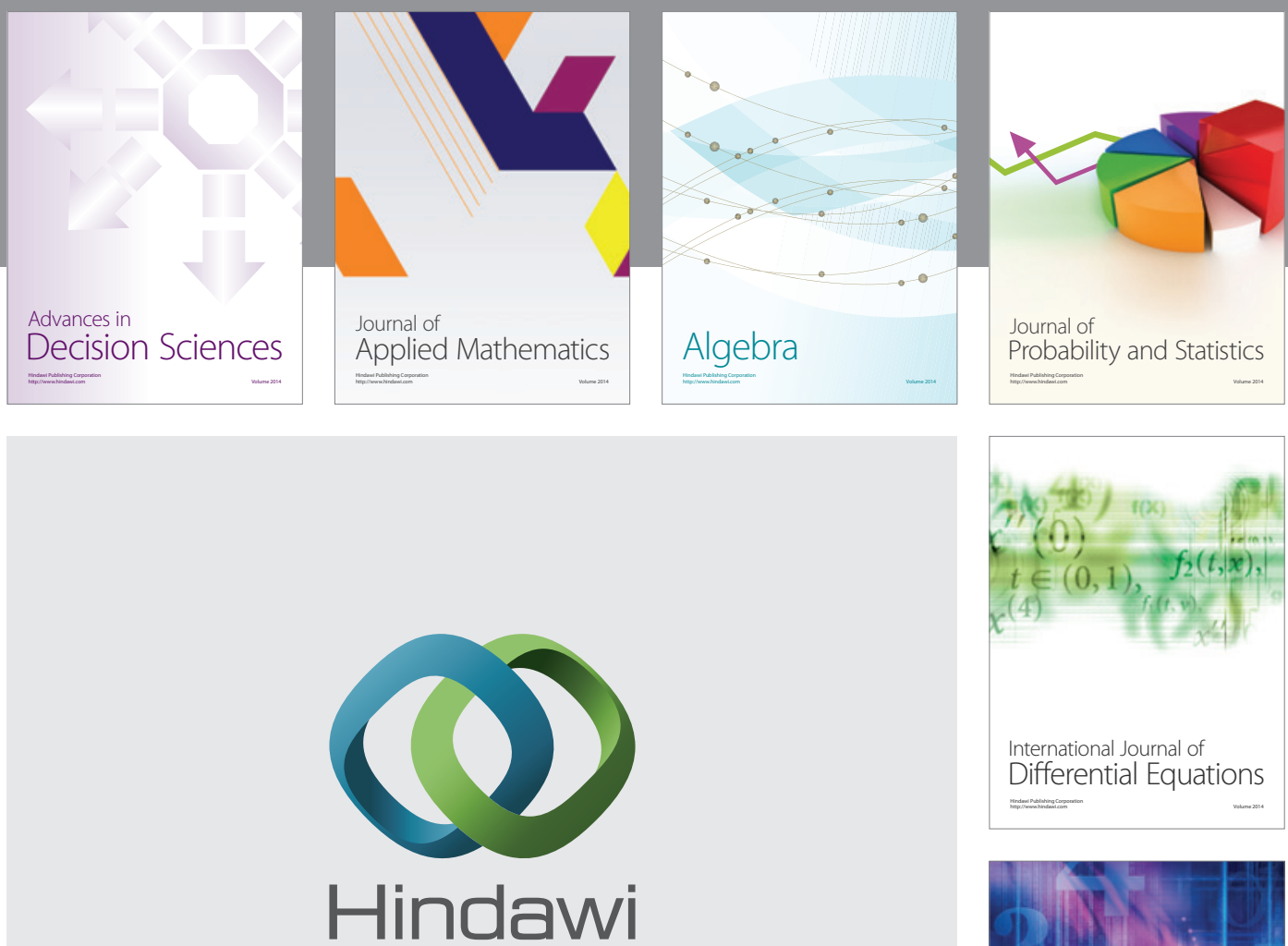

Submit your manuscripts at http://www.hindawi.com
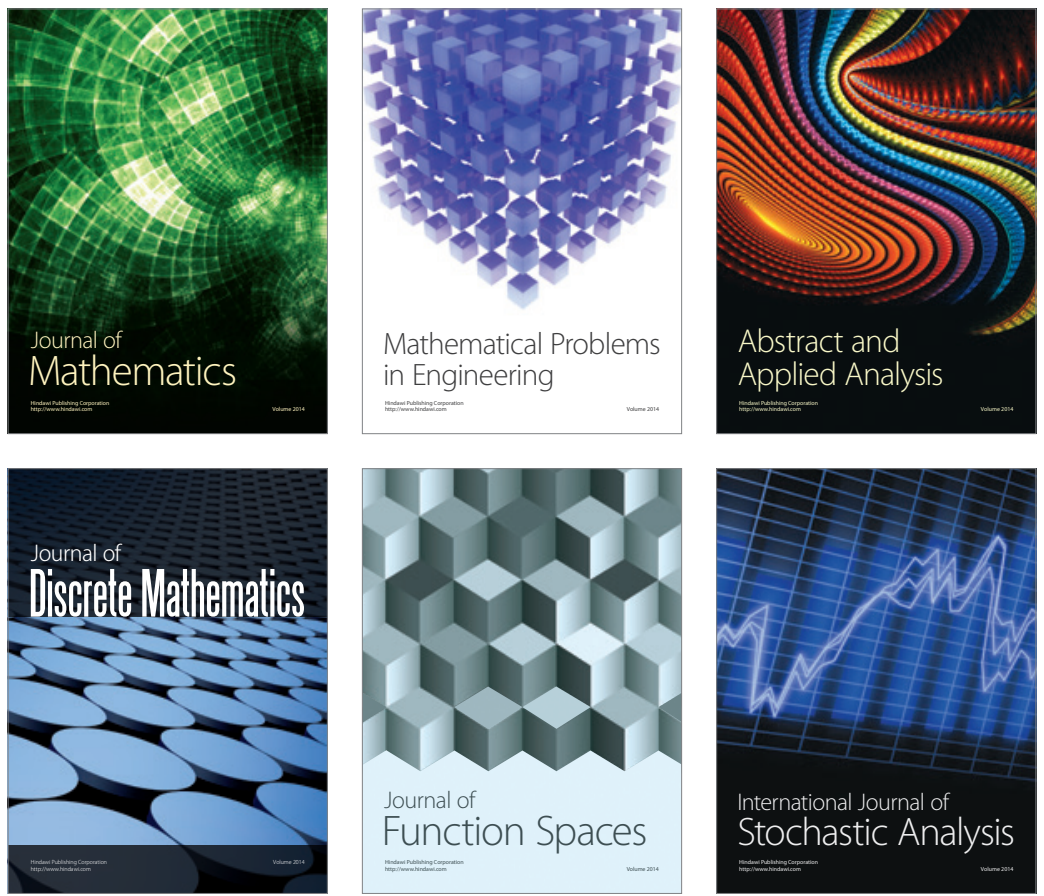

Journal of

Function Spaces

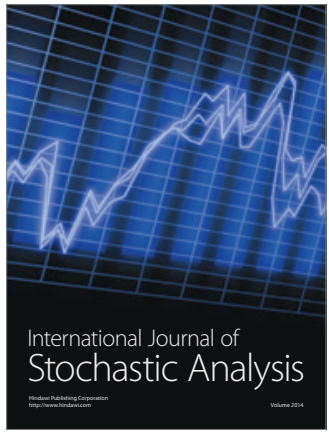

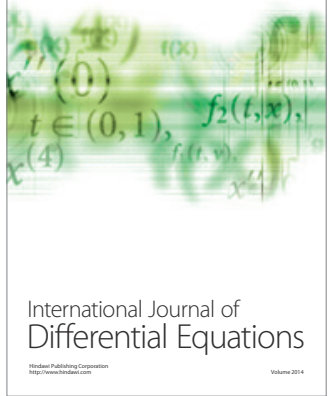
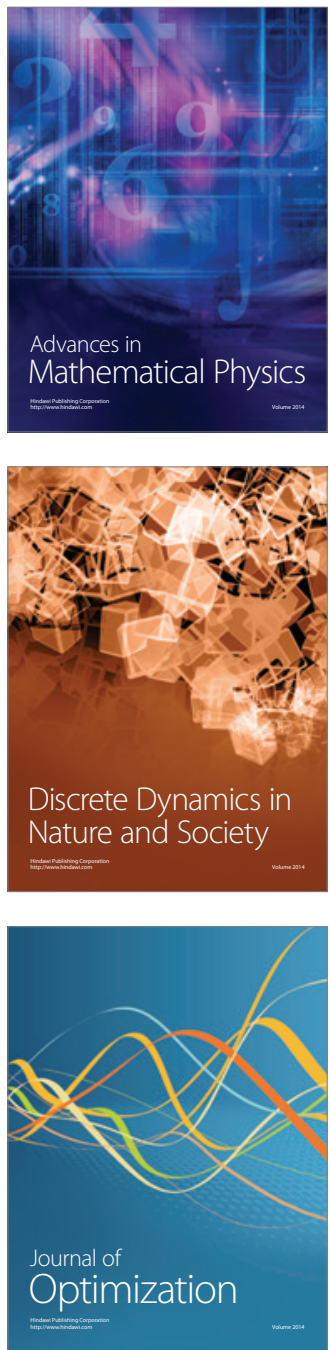